\title{
New algorithms for max restricted path consistency
}

\author{
Thanasis Balafoutis • Anastasia Paparrizou • \\ Kostas Stergiou • Toby Walsh
}

Published online: 29 June 2011

(C) Springer Science+Business Media, LLC 2011

\begin{abstract}
Max Restricted Path Consistency (maxRPC) is a local consistency for binary constraints that enforces a higher order of consistency than arc consistency. Despite the strong pruning that can be achieved, maxRPC is rarely used because existing maxRPC algorithms suffer from overheads and redundancies as they can repeatedly perform many constraint checks without triggering any value deletions. In this paper we propose and evaluate techniques that can boost the performance of maxRPC algorithms by eliminating many of these overheads and redundancies. These include the combined use of two data structures to avoid many redundant constraint checks, and the exploitation of residues to quickly verify the existence of supports. Based on these, we propose a number of closely related maxRPC algorithms. The first one, maxRPC 3 , has optimal $\mathrm{O}\left(e n d^{3}\right)$ time complexity, displays good performance when used stand-alone, but is expensive to apply during search. The second one, maxRPC $3^{r m}$, has $\mathrm{O}\left(e n^{2} d^{4}\right)$ time complexity, but a restricted version with $\mathrm{O}\left(e n d^{4}\right)$ complexity can be very efficient when used during search. The other algorithms are simple modifications of maxRPC $3^{r m}$. All algorithms have $\mathrm{O}(e d)$ space complexity when used stand-alone. However, maxRPC3 has $\mathrm{O}($ end $)$ space
\end{abstract}

This paper is an extended version of [1] that appeared in the proceedings of CP-2010.

T. Balafoutis

Department of Information and Communication Systems Engineering, University of the Aegean, University Hill, Administration Bldg., 81100,

Mytilene, Lesvos, Greece

A. Paparrizou $\cdot$ K. Stergiou $(\varangle)$

Department of Informatics and Telecommunications Engineering, University of Western Macedonia, Karamanli \& Lygeris, 50100 Kozani, FL, Greece e-mail: kstergiou@uowm.gr

A. Paparrizou

e-mail: apaparrizou@uowm.gr

T. Walsh

NICTA, University of New South Wales, Sydney, NSW 2052, Australia 
complexity when used during search, while the others retain the $\mathrm{O}(e d)$ complexity. Experimental results demonstrate that the resulting methods constantly outperform previous algorithms for maxRPC, often by large margins, and constitute a viable alternative to arc consistency on some problem classes.

Keywords Constraint propagation • Binary constraints • Local consistency

\section{Introduction}

Max Restricted Path Consistency (maxRPC) is a strong domain filtering consistency for binary constraints introduced in 1997 by Debruyne and Bessiere [14]. maxRPC achieves a stronger level of local consistency than arc consistency (AC), and in [15] it was identified, along with singleton AC (SAC), as a promising alternative to AC. Although SAC has received considerable attention since, maxRPC has been comparatively overlooked. Fewer new algorithms have been proposed and their experimental evaluation has been very limited. In this paper we propose new algorithms for maxRPC and evaluate them empirically on a wide range of problems.

The basic idea of maxRPC is to delete any value $a$ of a variable $x$ that has no arc consistency (AC) or path consistency (PC) support in a variable $y$ that is constrained with $x$. A value $b$ is an AC support for $a$ if the two values are compatible, and it is also a PC support for $a$ if this pair of values is path consistent. A pair of values $(a, b)$ is path consistent iff for every third variable there exists at least one value, called a PC witness, that is compatible with both $a$ and $b$.

The first algorithm for maxRPC was proposed in [14], and two more algorithms have been proposed since then [18, 28]. The algorithms of [14] and [28] have been evaluated on random problems only, while the algorithm of [18] has not been experimentally evaluated at all. Despite achieving stronger pruning than $\mathrm{AC}$, existing maxRPC algorithms suffer from overhead and redundancies as they can repeatedly perform many constraint checks without triggering any value deletions. These constraint checks occur when a maxRPC algorithm searches for an AC support for a value and when, having located one, it checks if it is also a PC support by looking for PC witnesses in other variables. As a result, the use of maxRPC during search often slows down the search process considerably compared to AC, despite the savings in search tree size.

In this paper we propose techniques to improve the applicability of maxRPC by eliminating some of these redundancies while keeping a low space complexity. We also investigate approximations of maxRPC that only make slightly fewer value deletions in practice, while being significantly faster. We first demonstrate that we can avoid many redundant constraint checks and speed up the search for AC and PC supports through the careful and combined application of two data structures already used by maxRPC and AC algorithms [10, 18, 22, 23, 28]. Based on this, we propose a coarse-grained maxRPC algorithm called maxRPC 3 with optimal O $\left(e n d^{3}\right)$ time complexity. This algorithm displays good performance when used stand-alone (e.g. for preprocessing), but is expensive to apply during search. We then propose another maxRPC algorithm, called maxRPC $3^{r m}$. This algorithm has $\mathrm{O}\left(e n^{2} d^{4}\right)$ time complexity, but a restricted version with $\mathrm{O}\left(e n d^{4}\right)$ complexity can be very efficient when used during search through the use of residues. Both algorithms have $\mathrm{O}(e d)$ 
space complexity when used stand-alone. However, maxRPC3 has $\mathrm{O}($ end $)$ space complexity when used during search, while maxRPC $3^{r m}$ retains the $\mathrm{O}(e d)$ complexity.

We further investigate the use of residues to improve the performance of maxRPC filtering during search. To be precise, we adapt ideas from [23] to obtain two variants of the maxRPC $3^{r m}$ algorithm. The first one achieves a better time complexity but is inferior to maxRPC $3^{r m}$ in practice, while the second one exploits in a simple way information obtained in the initialization phase of maxRPC $3^{r m}$ to achieve competitive performance.

Similar algorithmic improvements can be applied to light maxRPC (lmaxRPC), an approximation of maxRPC [28]. This achieves a lesser level of consistency compared to maxRPC, but still stronger than AC, and is more cost-effective when used during search. Experiments confirm that lmaxRPC is indeed a considerably better option than maxRPC when used throughout search. We also propose a number of heuristics that can be used to order the searches for PC supports and witnesses during the execution of a coarse-grained maxRPC algorithm, and in this way potentially save constraint checks.

Finally, we make a detailed experimental evaluation of new and existing algorithms on various problem classes. This is the first wide experimental study of algorithms for maxRPC and its approximations on benchmark non-random problems. We ran experiments with maxRPC algorithms under both a 2-way and a $d$-branching scheme. Results show that our methods constantly outperform existing algorithms, often by large margins, especially when 2-way branching is used. When applied during search our best method offers up to one order of magnitude reduction in constraint checks, while cpu times are improved up to three times compared to the best existing algorithm. In addition, these speed-ups enable a search algorithm that applies lmaxRPC to compete with or outperform MAC on some problems. Finally, we explore a simple hybrid propagation scheme where AC and maxRPC are interleaved under 2-way branching. Results demonstrate that instantiations of this scheme offer an efficient alternative to the application of a fixed propagation method (either AC or maxRPC) throughout search.

At this point we need to note that research on higher-order consistencies for binary constraints is not only motivated by the need to solve binary CSPs faster. Although there are some real problems that can be modeled using binary constraints only (e.g. frequency assignment problems), most real-life problems involve non-binary constraints. However, binary constraints still play an important role in Constraint Programming. First of all, some real problems include binary as well as non-binary constraints. Since applying higher-order consistencies on binary constraints is generally cheaper, such problems may benefit from applying a strong consistency on their binary part. Second, there are certain global constraints that can be reformulated as collections of binary or other low-arity constraints (decomposition methods), and indeed this is the approach taken by some solvers [9, 25]. Maintaining a strong local consistency on these reformulations may reduce the overall resolution time. Finally, as past experience has shown, research on binary constraints can inspire corresponding work on non-binary ones.

The remainder of this paper is structured as follows. Section 2 reviews background information on CSPs and related work on maxRPC algorithms. Section 3 presents two new algorithms, maxRPC $3, \operatorname{maxRPC} 3^{r m}$ and their corresponding approximations, and analyzes their complexities. Section 4 discusses the further exploitation of 
residues on two variations of the maxRPC $3^{r m}$ algorithm. Section 5 discusses heuristics for (1)maxRPC algorithms, and Section 6 presents our experimental results on benchmark problems. Finally, Section 7 concludes and discusses possible directions for future work.

\section{Background and related work}

A Constraint Satisfaction Problem (CSP) is defined as a tuple $(X, D, C)$ where: $X=$ $\left\{x_{1}, \ldots, x_{n}\right\}$ is a set of $n$ variables, $D=\left\{D^{0}\left(x_{1}\right), \ldots, D^{0}\left(x_{n}\right)\right\}$ is a set of initial domains, one for each variable, with maximum cardinality $d$, and $C=\left\{c_{1}, \ldots, c_{e}\right\}$ is a set of $e$ constraints. Each constraint $c$ is a pair $(\operatorname{var}(c), \operatorname{rel}(c))$, where $\operatorname{var}(c)=\left\{x_{1}, \ldots, x_{m}\right\}$ is an ordered subset of $X$, and $\operatorname{rel}(c)$ is a subset of the Cartesian product $D^{0}\left(x_{1}\right) \times \ldots \times$ $D^{0}\left(x_{m}\right)$ that specifies the allowed combinations of values for the variables in $\operatorname{var}(c)$. In the following, a binary constraint $c$ with $\operatorname{var}(c)=\left\{x_{i}, x_{j}\right\}$ will be denoted by $c_{i, j}$, and $D\left(x_{i}\right)$ will denote the current domain of variable $x_{i}$.

Each tuple $\tau$ of a constraint $c$ is an ordered list of values $\left(a_{1}, \ldots, a_{m}\right)$ such that $a_{j} \in D^{0}\left(x_{j}\right), j=1, \ldots, m$. That is, a tuple $\tau \in \operatorname{rel}(c)$ is a combination of values for the variables in the scope of $\operatorname{rel}(c)$. Tuple $\tau$ satisfies constraint $c$ iff $\tau \in \operatorname{rel}(c)$. Tuple $\tau=\left(a_{1}, \ldots, a_{m}\right)$ is valid iff $a_{j} \in D\left(x_{j}\right)$, for $j=1, \ldots, m$. In words, a valid tuple is an assignment of values to the variables involved in the constraint such that none of these values has been removed from the domain of the corresponding variable.

The process which verifies whether a given tuple is allowed by (i.e. satisfies) a constraint $c$ is called a constraint check. A constraint $c$ can be either defined extensionally by explicitly giving $\mathrm{rel}(\mathrm{c})$, or (usually) intensionally by implicitly specifying rel (c) through a predicate or arithmetic function. A binary CSP is a CSP where each constraint involves at most two variables and is typically represented by a constraint graph where nodes correspond to variables and edges correspond to constraints. We assume that binary constraint checks are performed in constant time.

In a binary CSP, a value $a_{i} \in D\left(x_{i}\right)$ is arc consistent (AC) iff for every constraint $c_{i, j}$ there exists a value $a_{j} \in D\left(x_{j}\right)$ s.t. the pair of values $\left(a_{i}, a_{j}\right)$ satisfies $c_{i, j}$. In this case $a_{j}$ is called an $A C$-support of $a_{i}$. A variable is AC iff all its values are AC. A problem is AC iff there is no empty domain in $D$ and all the variables in $X$ are AC.

Complete algorithms for CSPs are based on exhaustive backtracking search interleaved with constraint propagation. Search is typically guided by variable and value ordering heuristics and makes use of a specific branching scheme like 2-way or $d$-way branching.

In the experiments presented in this paper we have used the $d o m / w d e g$ variable ordering heuristic which is one of the most efficient general-purpose heuristics for CSPs [12]. This heuristic assigns a weight to each constraint, initially set to one. Each variable is associated with a weighted degree (wdeg), which is the sum of the weights over all constraints involving the variable and at least another (unassigned) variable. The $\mathrm{dom} / \mathrm{wdeg}$ heuristic chooses the variable with minimum ratio of current domain size to weighted degree.

The two most widely used branching schemes are 2-way and $d$-way. In 2-way branching, after a variable $x$ is chosen and a value $a_{i} \in D(x)$ is selected, two branches are created [26]. In the left branch $a_{i}$ is assigned to $x$, namely the constraint $x=a_{i}$ is added to the problem and is propagated. In the right branch the constraint $x \neq a_{i}$ is 
added to the problem and is propagated. If the left branch fails and the propagation of $x \neq a_{i}$ succeeds then any variable can be selected next (not necessarily $x$ ). If both branches fail then the algorithm backtracks. In $d$-way branching, after variable $x$ is selected, $d$ branches are built, each one corresponding to one of the $d$ possible value assignments of $x$. If the branch corresponding to assignment $x=a_{i}$ fails, the next available value assignment to $x$ is tried (next branch), and so on. If all $d$ branches fail then the algorithm backtracks. In Section 6 we report results from experiments with both branching schemes.

\section{1 maxRPC}

Several local consistencies stronger than AC have been proposed in the literature. Domain filtering consistencies are especially interesting because they only filter values from domains and as a result they do not alter either the constraint graph by adding new constraints, or the constraints' relations by removing inconsistent tuples [15]. Examples of domain filtering consistencies for binary constraints include restricted path consistency [4], path inverse consistency and neighborhood inverse consistency [16], max restricted path consistency [14], and singleton arc consistency [15]. The most famous local consistency that is not cast as domain filtering is path consistency. When enforced, path consistency can remove inconsistent 2-tuples from binary relations and/or introduce new binary constraints. Another recent example of such a local consistency is dual consistency [21].

The detailed study of domain filtering consistencies given in [15] concluded that maxRPC and SAC are promising strong consistencies that could be viable alternatives to AC. SAC, which is stronger than maxRPC, has received considerable attention since, and significant progress has been made regarding new SAC algorithms $[3,6,7,20]$. However, there is no concrete evidence yet that SAC can be cost-effective when maintained during search.

maxRPC was introduced in [14] as an extension to Restricted Path Consistency (RPC). A binary CSP is RPC iff it is AC and for each constraint $c_{i, j}$ and each value $a_{i} \in D\left(x_{i}\right)$ that has a single AC-support $a_{j} \in D\left(x_{j}\right)$, the pair of values $\left(a_{i}, a_{j}\right)$ is path consistent (PC) [4]. A pair of values $\left(a_{i}, a_{j}\right)$ is PC iff for any third variable $x_{k}$ there exists a value $a_{k} \in D\left(x_{k}\right)$ s.t. $a_{k}$ is an AC-support of both $a_{i}$ and $a_{j}$. In this case we say that $a_{j}$ is a $P C$-support of $a_{i}$ in $x_{j}$ and $a_{k}$ is a $P C$-witness for the pair $\left(a_{i}, a_{j}\right)$ in $x_{k}$.

A value $a_{i} \in D\left(x_{i}\right)$ is max restricted path consistent (maxRPC) iff it is AC and for each constraint $c_{i, j}$ there exists a value $a_{j} \in D\left(x_{j}\right)$ that is an AC-support of $a_{i}$ s.t. the pair of values $\left(a_{i}, a_{j}\right)$ is path consistent [14]. A variable is maxRPC iff all its values are maxRPC. A problem is maxRPC iff there is no empty domain in $D$ and all variables in $X$ are maxRPC.

A local consistency related to maxRPC is Path Inverse Consistency (PIC) [16]. A value $a_{i} \in D\left(x_{i}\right)$ is PIC iff for all $x_{j}, x_{k} \in X$, s.t. $x_{i} \neq x_{j} \neq x_{k} \neq x_{i}$, there exist $b_{j} \in$ $D\left(x_{j}\right)$ and $d_{k} \in D\left(x_{k}\right)$, s.t. the assignments $\left(x_{i}, a_{i}\right),\left(x_{j}, b_{j}\right)$ and $\left(x_{k}, d_{k}\right)$ satisfy the constraints between the three variables. Less formally, $a_{i}$ is PIC iff it is AC and it can be extended to all the 3-cliques of variables containing $x_{i}$. A variable is PIC iff all its values are PIC. A problem is PIC iff there is no empty domain in $D$ and all variables in $X$ are PIC.

A theoretical analysis and experimental results presented in [15] demonstrated that maxRPC is more efficient compared to RPC and PIC. Hence it was identified as 
a promising alternative to AC. This is why we focus on this local consistency in this paper.

Three algorithms for achieving maxRPC have been proposed in the literature so far. The first one, called maxRPC1 [14], is a fine-grained algorithm based on $\mathrm{AC}-6$ [5] and has optimal $\mathrm{O}\left(e n d^{3}\right)$ time complexity and $\mathrm{O}($ end $)$ space complexity. The second algorithm, called maxRPC2 [18], is a coarse-grained algorithm that uses ideas similar to those used by the AC algorithm AC2001/3.1 to achieve $\mathrm{O}\left(\right.$ end $\left.^{3}\right)$ time and $\mathrm{O}(e d)$ space complexity at the cost of some redundant checks compared to maxRPC1. The third algorithm, maxRPC ${ }^{r m}$ [28], is a coarse-grained algorithm based on $\mathrm{AC}^{\mathrm{rm}}$ [22]. The time and space complexities of maxRPC $C^{r m}$ are $\mathrm{O}\left(e n^{2} d^{4}\right)$ and $\mathrm{O}(e n d)$. Note that in [28] the complexities are given as $\mathrm{O}\left(e g+e d^{3}+c s d^{4}\right)$ and $\mathrm{O}(e d+c d)$, where $c$ is the number of 3-cliques, $g$ is the maximum degree of a variable and $s$ is the maximum number of 3-cliques that share the same single constraint in the constraint graph. Considering that $c$ is $\mathrm{O}(e n)$ and $s$ is $\mathrm{O}(n)$, we can derive the complexities for maxRPC ${ }^{r m}$ given here. This algorithm has a higher time complexity than the other two, but it has some advantages compared to them because of its lighter use of data structures during search (this is explained below and in Section 3.2). Finally, maxRPCEn1 is a fine-grained algorithm closely related to maxRPC1 [13]. This algorithm is based on $\mathrm{AC}-7$ [8] and achieves maxRPCEn, a local consistency stronger than maxRPC.

Among the three algorithms maxRPC2 seems to be the most promising for standalone use as it has a better time and space complexity than maxRPC ${ }^{r m}$ without requiring heavy data structures or complex implementation as maxRPC1 does. On the other hand, maxRPC ${ }^{r m}$ can be better suited for use during search as it avoids the costly maintainance of data structures as explained below.

Central to maxRPC2 is the LastPC data structure, as we call it here. For each constraint $c_{i, j}$ and each value $a_{i} \in D\left(x_{i}\right), \operatorname{Last} P C_{x_{i}, a_{i}, x_{j}}$ gives the most recently discovered PC-support of $a_{i}$ in $D\left(x_{j}\right)$. maxRPC2 maintains this data structure incrementally. This means that a copy of LastPC is made when moving forward during search (i.e. after a successfully propagated variable assignment) and Last PC is restored to its previous state after a failed variable assignment (or value removal in the case of 2-way branching). Data structures with such a property are often referred to as backtrackable. Their use implies an increased space complexity as copies need to be made while search progresses down a branch. On a brighter note, since Last PC is backtrackable, maxRPC2 displays the following behavior: When looking for a PCsupport for $a_{i}$ in $D\left(x_{j}\right)$, it first checks if $\operatorname{Last} P C_{x_{i}, a_{i}, x_{j}}$ is valid. If it is not, it searches for a new PC-support starting from the value immediately after $\operatorname{Last}_{P} C_{x_{i}, a_{i}, x_{j}}$ in $D\left(x_{j}\right)$. In this way a good time complexity bound is achieved.

On the other hand, maxRPC ${ }^{r m}$ uses a data structure similar to Last PC to store residual supports or simply residues, i.e. supports that have been discovered during execution and stored for future use, but does not maintain this structure incrementally (it only needs one copy). Therefore, no additional actions need to be taken (copying or restoration) when moving forward or after a fail. Such data structures are often referred to as backtrack-stable. When looking for a PC-support for $a_{i}$ in

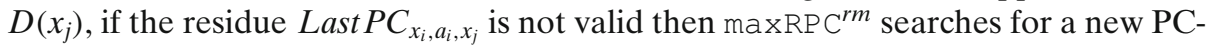
support from scratch in $D\left(x_{j}\right)$. This results in higher time complexity, but crucially does not require costly maintainance of Last PC during search. The algorithm also makes use of residues for the PC-witnesses found in every third variable for each 
pair $\left(a_{i}, a_{j}\right)$. These are stored in a data structure with an $\mathrm{O}(e n d)$ space complexity. The initialization of this structure causes an extra overhead which can be significant on very large problems.

A major overhead of both maxRPC2 and maxRPC ${ }^{r m}$ is the following. When searching for a PC-witness for a pair of values $\left(a_{i}, a_{j}\right)$ in a third variable $x_{k}$, they always start the search from scratch, i.e. from the first available value in $D\left(x_{k}\right)$. As these searches can be repeated many times during search, there can be many redundant constraint checks. In contrast, maxRPC1 manages to avoid searching from scratch through the use of an additional data structure. This saves many constraint checks, albeit resulting in $\mathrm{O}($ end $)$ space complexity and requiring costly maintainance of this data structure during search. The algorithms we describe below largely eliminate these redundant constraint checks with lower space complexity, and in the case of maxRPC $3^{r m}$ with only light use of data structures.

\section{New algorithms for maxRPC}

We first recall the basic ideas of algorithms maxRPC2 and maxRPC ${ }^{r m}$ as described in [18] and [28]. Both algorithms use a propagation list $Q$ where variables whose domain is pruned are added. Once a variable $x_{j}$ is removed from $Q$ all neighboring variables are revised to delete any values that are no longer maxRPC. For any value $a_{i}$ of such a variable $x_{i}$ there are three possible reasons for deletion:

- The first is when $a_{i}$ no longer has an AC-support in $D\left(x_{j}\right)$.

- The second, which we call PC-support loss hereafter, is when the unique PCsupport $a_{j} \in D\left(x_{j}\right)$ for $a_{i}$ has been deleted.

- The third, which we call $P C$-witness loss hereafter, is when the unique PC-witness $a_{j} \in D\left(x_{j}\right)$ for the pair $\left(a_{i}, a_{k}\right)$, where $a_{k}$ is the unique PC-support for $a_{i}$ on some variable $x_{k}$, has been deleted.

If any of the above cases occurs then value $a_{i}$ is no longer maxRPC.

We now present the pseudocodes for the new maxRPC algorithms, maxRPC 3 and maxRPC $3^{r m}$. Both algorithms utilize data structures Last $P C$ and Last AC which have the following functionalities: For each constraint $c_{i, j}$ and each value $a_{i} \in D\left(x_{i}\right)$, Last $P C_{x_{i}, a_{i}, x_{j}}$ and Last $A C_{x_{i}, a_{i}, x_{j}}$ point to the most recently discovered PC and AC supports of $a_{i}$ in $D\left(x_{j}\right)$ respectively. Initially, all Last $P C$ and Last $A C$ pointers are set to a special value NIL, considered to precede all values in any domain. As will be explained, algorithm maxRPC3 updates the Last PC and Last AC structures incrementally like maxRPC2 and $\mathrm{AC} 2001 / 3.1$ respectively do. In contrast, algorithm maxRPC $3^{r m}$ uses these structures as residues like maxRPC ${ }^{r m}$ and $\mathrm{AC}^{r m}$ do.

\section{1 maxRPC3}

The main part of maxRPC3 is described in Algorithm 1. Since maxRPC3 is coarsegrained, it uses a propagation list $Q$ (typically implemented as a queue) where variables that have their domain filtered are inserted. This may happen during initialization (explained below) or when PC-support or PC-witness loss is detected. When a variable $x_{j}$ is removed from $Q$, at line 4 , each variable $x_{i}$ constrained with $x_{j}$ 
must be checked for possible AC-support, PC-support or PC-witness loss. We now discuss the overall function of the algorithm before moving on to explain it in detail.

For each value $a_{i} \in D\left(x_{i}\right)$, Algorithm 1 first checks whether $a_{i}$ has suffered ACsupport or PC-support loss in $D\left(x_{j}\right)$ by calling function checkPCsupLoss, provided that $\operatorname{Last} P C_{x_{i}, a_{i}, x_{j}}$ is not valid anymore (line 7). This function, which will be explained in detail below, returns false if no new PC-support exists for $a_{i}$ in $D\left(x_{j}\right)$ and as a result $a_{i}$ is deleted (line 8). If $a_{i}$ is not deleted, either because Last $P C_{x_{i}, a_{i}, x_{j}}$ is still valid or because a new PC-support for $a_{i}$ has been found in $D\left(x_{j}\right)$, then possible PC-witness loss is examined by calling function checkPCwitLoss (line 11). If this function returns false, then $a_{i}$ is deleted (line 12). If a value is deleted from $D\left(x_{i}\right)$ then $x_{i}$ is inserted to $Q$ (lines 9 and 13). After deleting values from the domain of a variable, the algorithm checks whether the domain is empty (line 14). If so, the algorithm returns FAILURE.

An important remark about Algorithm 1 is the following. Assuming a value $a_{i}$ has been examined in lines $6-13$ and has not been deleted, then this does not necessarily mean that $a_{i}$ is maxRPC. Indeed there is the possibility that $\operatorname{Last}_{P} C_{x_{i}, a_{i}, x_{j}}$ is valid but the last PC-witness of the pair $\left(a_{i}\right.$, Last $\left.P C_{x_{i}, a_{i}, x_{j}}\right)$ in some variable $x_{k}$ has been deleted. Hence, if Last $P C_{x_{i}, a_{i}, x_{j}}$ is the last PC-support of $a_{i}$ in $D\left(x_{j}\right)$ then $a_{i}$ is not maxRPC. Such a situation will be identified at some point during the execution of the algorithm once $x_{k}$ is removed from $Q$ and its neighboring variables are examined. This guarantees the algorithm's completeness as will be further explained in Section 3.4.

The initialization step of maxRPC3 (Function 2) is a brute-force function, where each value $a_{i}$ of each variable $x_{i}$ is checked for being maxRPC. This is done by iterating through the variables constrained with $x_{i}$ and looking for a PC-support for $a_{i}$ in their domains. For each such variable $x_{j}$ and value $a_{j} \in D\left(x_{j}\right)$, we first check if the pair $\left(a_{i}, a_{j}\right)$ is arc consistent by calling function is Consistent at line 6 . isConsistent returns true if $\left(a_{i}, a_{j}\right)$ satisfies the constraint, meaning that $a_{j} \mathrm{AC}$-supports $a_{i}$. In this case Last $A C_{x_{i}, a_{i}, x_{j}}$ is set to $a_{j}$ (line 7). If $a_{j}$ is verified as an AC-support of $a_{i}$, we examine if it is also a PC-support by calling function searchPCwit. If searchPCwit returns true (detailed analysis follows below), then $\operatorname{Last} P C_{x_{i}, a_{i}, x_{j}}$ is set to $a_{j}$ (line 10), since $a_{j}$ is the most recently found PC-support for $a_{i}$. Line 11 will be explained below when algorithm maxRPC $3^{r m}$ is presented. Then, the next variable constrained with $x_{i}$ will be considered, and so on.

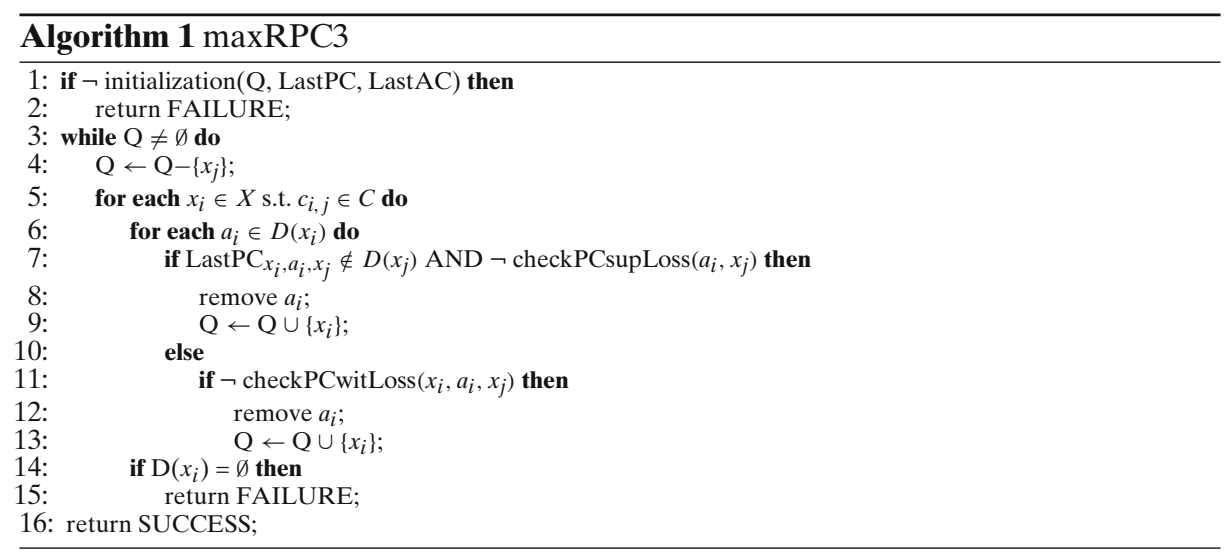




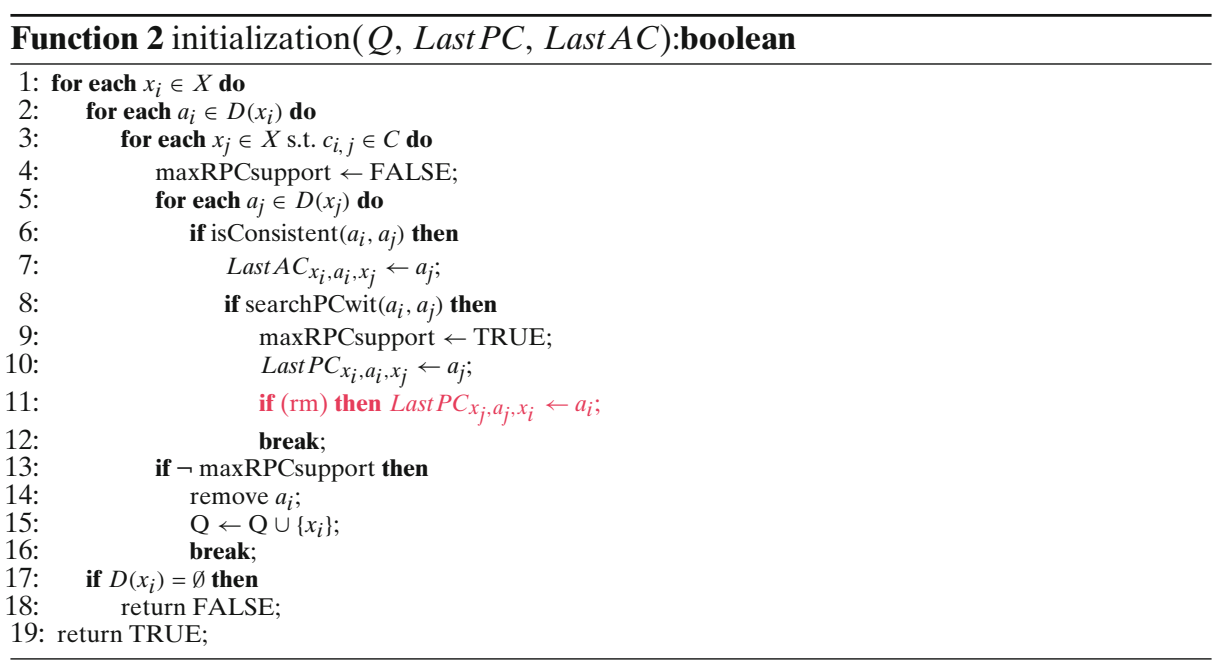

If there is no AC-support in $D\left(x_{j}\right)$ for $a_{i}$ or none of the AC-supports is a PCsupport, then $a_{i}$ will be removed at line 14 and $x_{i}$ will be added to queue $Q$. Eventually, $a_{i}$ is established to be maxRPC when a PC-support is found in each $D\left(x_{j}\right)$, where $x_{j}$ has a constraint with $x_{i}$. Finally, if function initialization causes an empty domain (line 17), then maxRPC 3 returs FAILURE in line 2 of Algorithm 1 . Note that initilization is called only when maxRPC3 is used stand-alone (e.g. for preprocessing) and not during search, as in this case $Q$ is initialized with the variable of the latest decision.

Assuming the initialization phase succeeded, the propagation list $Q$ will include those variables that have their domain filtered. The main part of maxRPC3 (Algorithm 1) starts when a variable $x_{j}$ is extracted from $Q$ (line 4$)$ in order to determine whether a neighbouring variable $\left(x_{i}\right)$ has suffered PC-support or PC-witness loss due to the filtering of the extracted variable's domain. These checks are implemented by calling functions checkPCsupLoss and checkPCwitLoss, at lines 7 and 11 of Algorithm 1, for each value $a_{i} \in D\left(x_{i}\right)$. If value Last $P C_{x_{i}, a_{i}, x_{j}}$ is still in $D\left(x_{j}\right)$ line 7 , then a possible PC-support has been immediately located (the PC-support will be established later as explained in the remark about the algorithm given above) and checkPCsupLoss is not called. In the opposite case where Last $P C_{x_{i}, a_{i}, x_{j}}$ is not valid, checkPCsupLoss is called to search for a new PC-support in $D\left(x_{j}\right)$.

\subsubsection{Checking for PC-support loss}

Function checkPCsupLoss (Function 3) takes advantage of the Last PC and Last AC pointers to avoid starting the search for PC-support from scratch. Specifically, we

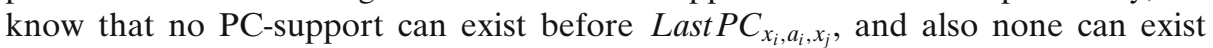

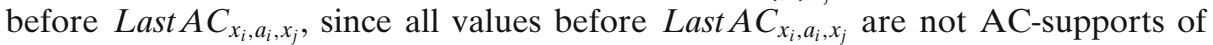
$a_{i}$. Lines 1-4 in checkPCsupLoss take advantage of these to locate the appropriate starting value $b_{j}$. Note that maxRPC2 always starts the search for a PC-support from the value after $\operatorname{Last}_{P} C_{x_{i}, a_{i}, x_{j}}$ and thus may perform redundant constraint checks.

For every value $a_{j} \in D\left(x_{j}\right)$, starting with $b_{j}$, we first check if it is an AC-support of $a_{i}$ by calling function is Consistent (line 6). If it is, then we can update Last $A C_{x_{i}, a_{i}, x_{j}}$ 


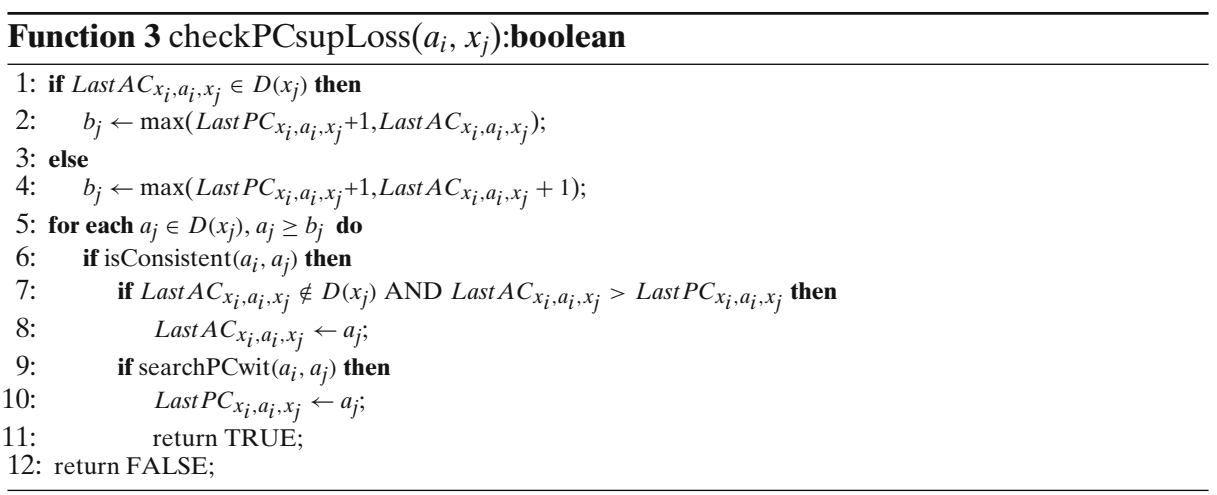

under a certain condition (lines 7-8). Specifically, if $L a s t A C_{x_{i}, a_{i}, x_{j}}$ was deleted from $D\left(x_{j}\right)$, then we can set $\operatorname{Last}_{A} A C_{x_{i}, a_{i}, x_{j}}$ to $a_{j}$ in case $\operatorname{Last} A C_{x_{i}, a_{i}, x_{j}}>\operatorname{Last} P C_{x_{i}, a_{i}, x_{j}}$. If $\operatorname{Last}_{A} C_{x_{i}, a_{i}, x_{j}} \leq \operatorname{Last} P C_{x_{i}, a_{i}, x_{j}}$ then we cannot do this update, as there may be AC-supports for $a_{i}$ between Last $A C_{x_{i}, a_{i}, x_{j}}$ and $\operatorname{Last} P C_{x_{i}, a_{i}, x_{j}}$ in the lexicographical ordering. We then move on to verify the path consistency of $\left(a_{i}, a_{j}\right)$ through function searchPCwit (line 9). If no PC-support for $a_{i}$ is found in $D\left(x_{j}\right)$, checkPCsupLoss will return false, $a_{i}$ will be deleted and $x_{i}$ will be added to $Q$ in Algorithm 1. Otherwise, Last $P C_{x_{i}, a_{i}, x_{j}}$ is set to the discovered PC-support $a_{j}$ (line 10).

Function searchPCwit (Function 4) checks if a pair of values $\left(a_{i}, a_{j}\right)$ is PC by doing the following for each variable $x_{k}$ constrained with $x_{i}$ and $x_{j}$. ${ }^{1}$ First, taking advantage of the Last $A C$ pointers, it makes a quick check in constant time which, if successful, can save searching in the domain of $x_{k}$. To be precise, it checks if Last $A C_{x_{i}, a_{i}, x_{k}}$ is valid and Last $A C_{x_{i}, a_{i}, x_{k}}$ equals $\operatorname{Last} A C_{x_{j}, a_{j}, x_{k}}$, or if $\operatorname{Last} A C_{x_{i}, a_{i}, x_{k}}$ is valid and consistent with $a_{j}$ or if $\operatorname{Last} A C_{x_{j}, a_{j}, x_{k}}$ is valid and consistent with $a_{i}$ (line 3 ). The first part of the disjunction is of practical importance only, since if it is true, then the second part will necessarily also be true and the condition will be verified. However, including the first part of the condition saves constraint checks, and this reflects on run times in certain problems.

If one of these conditions holds then we have found a PC-witness for $\left(a_{i}, a_{j}\right)$ without searching in $D\left(x_{k}\right)$ and we move on to the next variable constrained with $x_{i}$ and $x_{j}$. Note that neither maxRPC2 nor maxRPC ${ }^{r m}$ can do this check as they do not have the Last $A C$ structure. In contrast, algorithm maxRPCEn 1 is able to do such reasoning. Experimental results in Section 6 demonstrate that these simple conditions of line 3 can eliminate a very large number of redundant constraint checks.

If none of the conditions in line 3 of Function 4 holds, searching for a new PCwitness in $D\left(x_{k}\right)$ is necessary. This is done by first calling function searchACsup (Function 5), first with $\left(a_{i}, x_{k}\right)$ and then with $\left(a_{j}, x_{k}\right)$ as parameters. This function locates the lexicographically smallest AC-supports for $a_{i}$ and $a_{j}$ in $D\left(x_{k}\right)$. More precisely, searchACsup checks if the current Last $A C$ value exists in the corresponding domain (line 1 of Function 5), and if not it searches for a new AC-support after that (line 4). If it finds one, it updates Last $A C$ accordingly (line 6).

\footnotetext{
${ }^{1}$ Since AC is enforced by the maxRPC algorithm, we only need to consider variables that form a 3-clique with $x_{i}$ and $x_{j}$ [24].
} 


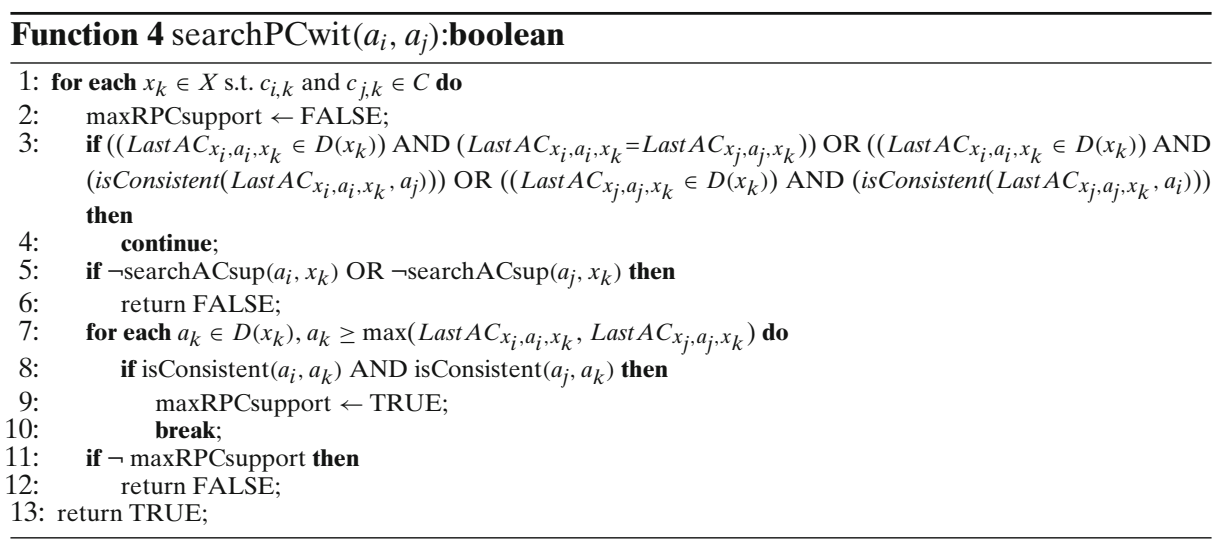

Then, going back to searchPCwit the search for a PC-witness starts from $b_{k}=$ $\max \left\{\operatorname{Last} A C_{x_{i}, a_{i}, x_{k}}\right.$, Last $\left.A C_{x_{j}, a_{j}, x_{k}}\right\}$ (line 7), exploiting the Last $A C$ structure to save redundant checks (a similar operation is performed by maxRPCEn1). This search looks for a value of $x_{k}$ that is compatible with both $a_{i}$ and $a_{j}$ (line 8). If no ACsupport is found for either $a_{i}$ or $a_{j}$ (in which cases searchACsup returns false) or no $\mathrm{PC}$-witness is located, then subsequently searchPCwit will also return false.

\subsubsection{Checking for PC-witness loss}

In maxRPC 3 , if value $a_{i}$ is not removed after checking for possible PC-support loss using checkPCsupLoss, function checkPCwitLoss (Function 6) is called to check for PC-witness loss. This is done by iterating over the variables that are constrained with both $x_{i}$ and $x_{j}$. For each such variable $x_{k}$, we first check if $a_{k}=\operatorname{Last} P C_{x_{i}, a_{i}, x_{k}}$ is still in $D\left(x_{k}\right)$ (line 3 ). If so then we verify if there is still a PC-witness in $D\left(x_{j}\right)$. As in function searchPCwit, taking advantage of the Last $A C$ pointers, we first make a quick check in constant time which, if successful, can save searching in the domain of $x_{j}$. That is, we check if $\operatorname{Last} A C_{x_{i}, a_{i}, x_{j}}$ is valid and $\operatorname{Last} A C_{x_{i}, a_{i}, x_{j}}$ equals $\operatorname{Last} A C_{x_{k}, a_{k}, x_{j}}$ or if $\operatorname{Last} A C_{x_{i}, a_{i}, x_{j}}$ is valid and consistent with $a_{k}$ or if $\operatorname{Last}_{A} A C_{x_{k}, a_{k}, x_{j}}$ is valid and consistent with $a_{i}$ (line 4). If none of these conditions holds then we search for a PCwitness starting from $b_{j}=\max \left\{\right.$ Last $A C_{x_{i}, a_{i}, x_{j}}$, Last $\left.A C_{x_{k}, a_{k}, x_{j}}\right\}$ (line 8), after checking the existence of AC-supports for $a_{i}$ and $a_{k}$ in $D\left(x_{j}\right)$, by calling searchACsup (line 7). Right here the procedure is quite similar to searchPCwit. If there is no AC-support in $D\left(x_{j}\right)$ for either $a_{i}$ or $a_{k}$ we avoid searching for a PC-witness in $D\left(x_{j}\right)$ and move on

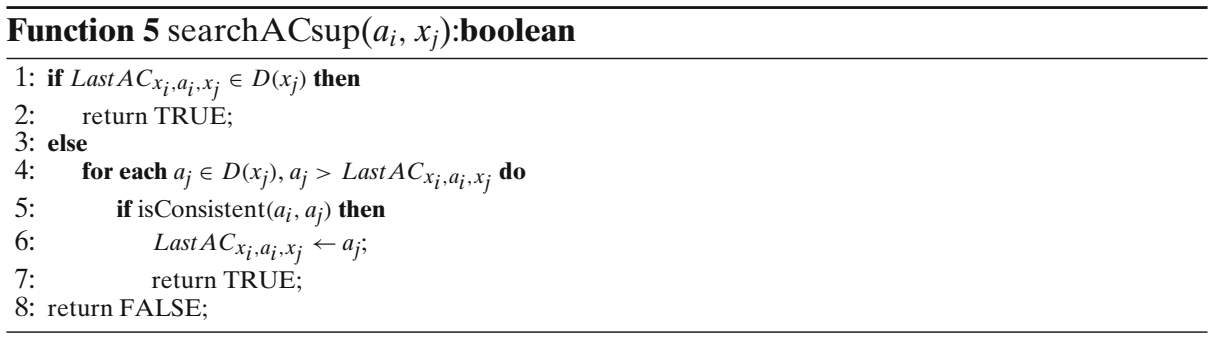




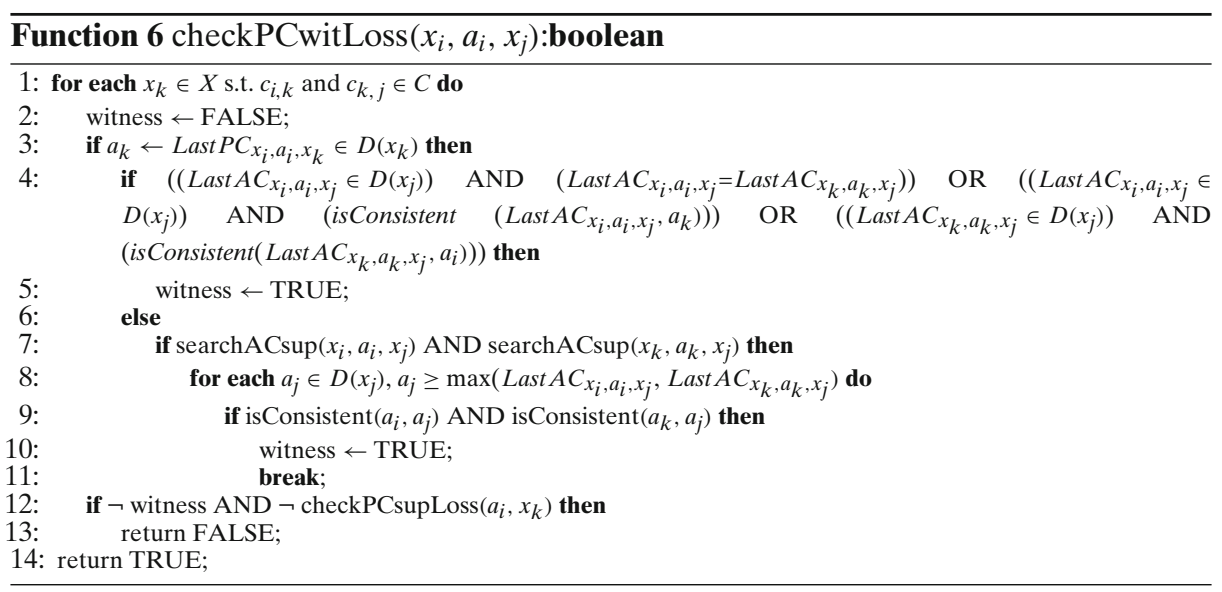

to seek a new PC-support for $a_{i}$ in $D\left(x_{k}\right)$. Note that maxRPC2 does not do the check of line 4 and always starts the search for a PC-witness from the first value in $D\left(x_{j}\right)$.

If Last $P C_{x_{i}, a_{i}, x_{k}}$ has been removed or the pair $\left(a_{i}, a_{k}\right)$ has no PC-witness in $D\left(x_{j}\right)$, we search for a new PC-support for $a_{i}$ in $D\left(x_{k}\right)$ in line 12 by calling function checkPCsupLoss. Search starts at an appropriate value calculated taking advantage of Last $P C_{x_{i}, a_{i}, x_{k}}$ and Last $A C_{x_{i}, a_{i}, x_{k}}$ (lines 1-4 in Function 3). The procedure was explained above when describing checkPCsupLoss. If the search for a PC-support fails for any third variable $x_{k}$ then false will be returned, and in the main algorithm $a_{i}$ will be deleted and $x_{i}$ will be added to $Q$.

maxRPC3 terminates when $Q$ becomes empty, meaning that all values are maxRC, or, when a domain of some variable becomes empty, meaning that the problem is not consistent.

As observed above, when maxRPC3 is applied during search, the propagation list $Q$ is initialized with the variable at the current decision (assignment or value removal). If propagating a decision results in an empty domain, then both the Last $A C$ and LastPC data structures must be restored to their state prior to the decision.

\section{2 maxRPC3 ${ }^{r m}$}

maxRPC $3^{r m}$ is a coarse-grained maxRPC algorithm that exploits backtrack-stable data structures inspired from $A C 3^{r m}$ ( $\mathrm{rm}$ stands for multidirectional residues). Last $A C$ and Last $P C$ are not maintained incrementally as in maxRPC3, but are only used to store residues. As explained, a residue is a support which has been located and stored during the execution of the procedure that proves that a given value is $\mathrm{AC}$ or PC. The algorithm stores the most recently discovered AC (resp. PC) supports, but does not guarantee that any lexicographically smaller value is not an AC (resp. PC) support. Consequently, when we search for a new AC or PC support in a domain, we always start from scratch. Last $A C$ and Last $P C$ need not be restored after a failure; they can remain unchanged, hence a minimal overhead on the management of data.

Another difference with maxRPC 3 is that since maxRPC $3^{r m}$ handles Last PC only as a residue, it can exploit the bidirectionality of support. This means that when a 


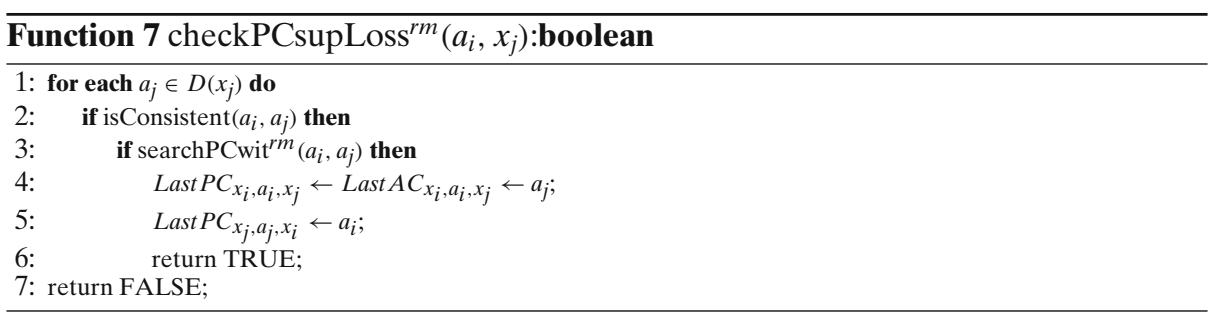

PC-support $a_{j} \in D\left(x_{j}\right)$ is located for a value $a_{i} \in D\left(x_{i}\right)$ then $a_{i}$ is a PC-support for $a_{j}$. As a result, we can assign Last $P C_{x_{i}, a_{i}, x_{j}}$ and Last $P C_{x_{j}, a_{j}, x_{i}}$ to $a_{j}$ and to $a_{i}$ respectively. Although the property of bidirectionality obviously also holds for AC-supports, we do not exploit this since experiments demonstrated that it does not offer any benefits in most cases. Moreover, Last AC is updated when a PC-support is found, since it is also the most recent AC-support found. This assignment may speed up subsequent searches for PC-witness as the conditions in line 3 of searchPCwit ${ }^{r m}$ and line 4 of checkPCwitLoss $^{r m}$ are more likely to be true.

We omit presenting the main algorithm for maxRPC $3^{r m}$ as it is the same as Algorithm 1 with the only difference being that we call checkPCsupLoss ${ }^{r m}$ and checkPCwitLoss $^{r m}$ instead of checkPCsupLoss and checkPCwitLoss respectively. When maxRPC $3^{r m}$ is used for preprocessing, the initialization function (Function 2) is called to initialize $Q$ and structures Last $A C$ and Last $P C$. The difference with maxRPC3 concerns the bidirectionality of PC-supports. If the auxilary boolean variable $r m$ is true, denoting the use of maxRPC $3^{r m}$ instead of maxRPC3, we initialize the Last $P C$ residue exploiting bidirectionality. To be precise, when a PC-support is found for $a_{i}$ in $D\left(x_{j}\right)$ we set $\operatorname{Last}_{P} C_{x_{i}, a_{i}, x_{j}}$ to $a_{j}$ and additionally $\operatorname{Last} P C_{x_{j}, a_{j}, x_{i}}$ to $a_{i}$ (line 11 of Function 2).

When a variable is extracted from $Q$, we first explore the case of PC-support loss by calling function checkPCsupLoss ${ }^{r m}$, after verifying that value $\operatorname{Last} P C_{x_{i}, a_{i}, x_{j}}$ is not in $D\left(x_{j}\right)$ anymore. checkPCsupLoss ${ }^{r m}$ (Function 7) searches for a new PCsupport starting from scratch (line 1). In contrast, maxRPC3 would start from $b_{j}=\max \left(\operatorname{Last} P C\left(x_{i}, a_{i}, x_{j}\right)\right.$, Last $\left.A C\left(x_{i}, a_{i}, x_{j}\right)\right)$ and maxRPC2 from the value after Last $P C_{x_{i}, a_{i}, x_{j}}$. When an AC-support $a_{j}$ is confirmed from isConsistent in line 2,

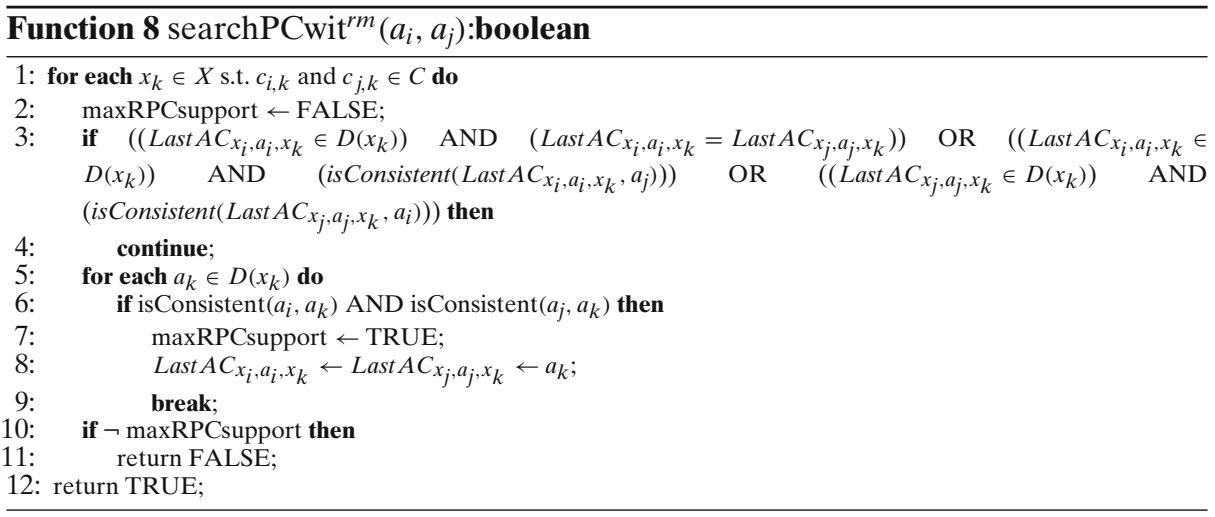




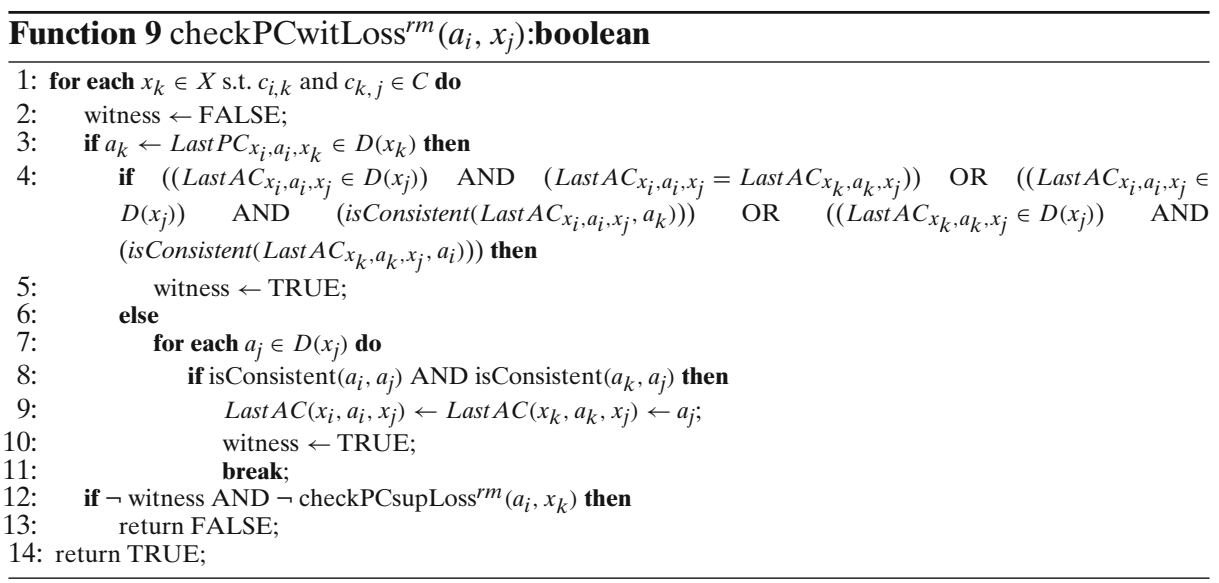

function searchPCwit ${ }^{r m}$ is called to determine if $a_{j}$ is also a PC-support for $a_{i}$. If searchPCwit ${ }^{r m}$ returns true, we assign Last $P C_{x_{i}, a_{i}, x_{j}}$ and Last $P C_{x_{j}, a_{j}, x_{i}}$ to $a_{j}$ and to $a_{i}$ respectively to exploit bidirectionality, and $\operatorname{Last} A C_{x_{i}, a_{i}, x_{j}}$ is set to $a_{j}$ (lines 4-5), since the discovered PC-support is also an AC-support.

Function searchPCwit ${ }^{r m}$ (Function 8) checks if a pair of values $\left(a_{i}, a_{j}\right)$ is PC by iterating over the variables $x_{k}$ constrained with $x_{i}$ and $x_{j}$. First, it checks the same conditions in line 3 as searchPCwit to locate, if possible, a PC-witness without searching. If none of these conditions holds, it searches for a new PC-support starting from the first value in $D\left(x_{k}\right)$ (line 5). If a PC-witness $a_{k}$ is found (line 7) then both residues, Last $A C_{x_{i}, a_{i}, x_{k}}$ and $\operatorname{Last} A C_{x_{j}, a_{j}, x_{k}}$, are set to $a_{k}$ as they are the most recently discovered AC-supports (line 8). If no PC-witness is found we have determined that the pair $\left(a_{i}, a_{j}\right)$ is not PC and as a result false will be returned and checkPCsupLoss ${ }^{r m}$ will move to check if the next available value in $D\left(x_{j}\right)$ is a PC-support for $a_{i}$.

In maxRPC $3^{r m}$, if value $a_{i}$ is not removed after checking for possible PC-support loss using checkPCsupLoss ${ }^{r m}$, function checkPCwitLoss ${ }^{r m}$ (Function 9) is called to check for PC-witness loss. This is done by iterating again, over the variables that are constrained with both $x_{i}$ and $x_{j}$. For each such variable $x_{k}$, we first check if $a_{k}=$ Last $P C_{x_{i}, a_{i}, x_{k}}$ remains in $D\left(x_{k}\right)$ (line 3 ) and if so, if any of the three conditions in line 4 is satisfied in order to avoid searching. In case each of these conditions fails, we search for a new PC-witness in $D\left(x_{j}\right)$ starting from the first value (line 7). For each value $a_{j} \in D\left(x_{j}\right)$, checkPCwitLoss ${ }^{r m}$ checks if it is compatible with $a_{i}$ and $a_{k}$ and moves the Last AC pointers accordingly (line 9), exploiting the bidirectionality of residues.

If Last $P C_{x_{i}, a_{i}, x_{k}}$ is not valid or the pair $\left(a_{i}, a_{k}\right)$ fails to find a PC-witness in $D\left(x_{j}\right)$, we search for a new PC-support for $a_{i}$ in $D\left(x_{k}\right)$ in line 12, by calling checkPCsupLoss ${ }^{r m}$. If the search for a PC-support fails then false will be returned (line 13), $a_{i}$ will be deleted, and $x_{i}$ will be added to $Q$ in the main algorithm.

\subsection{Light maxRPC}

Light maxRPC (lmaxRPC) is an approximation of maxRPC that only propagates the loss of AC-supports and not the loss of PC-witnesses [28]. That is, when removing a 
variable $x_{j}$ from $Q$, for each $a_{i} \in D\left(x_{i}\right)$, where $x_{i}$ is constrained with $x_{j}$, lmaxRPC only checks if there is a PC-support of $a_{i}$ in $D\left(x_{j}\right)$. This ensures that the obtained algorithm enforces a consistency property that is at least as strong as AC.

lmaxRPC is a procedurally defined local consistency, meaning that its description is tied to a specific maxRPC algorithm. Hence when applying this consistency a fixed point is dependent on the particularities of the specific algorithm used, like the order in which the algorithm processes revisions of variables/constraints, and the order in which values are processed and supports as seeked. Light versions of algorithms maxRPC3 and maxRPC $3^{r m}$, simply noted lmaxRPC3 and 1 maxRPC $3^{r m}$ respectively, can be obtained by omitting the call to the checkPCwitLoss (resp. checkPCwitLoss $^{\text {rm }}$ ) function (lines 10-13 of Algorithm 1). In a similar way, we can obtain light versions of algorithms maxRPC2 and maxRPC ${ }^{r m}$.

As already noted in [28], the light versions of different maxRPC algorithms may not be equivalent in terms of the pruning they achieve. To give an example, a bruteforce algorithm for lmaxRPC that does not use any of the data structures described here can achieve more pruning than algorithms 1 maxRPC2, ImaxRPC 3,1 maxRPC ${ }^{r m}$, and 1 maxRPC $3^{r m}$, albeit being much slower in practice. This is because when looking for a PC-support for a value $a_{i} \in D\left(x_{i}\right)$ in a variable $x_{j}$, the brute-force algorithm will always search in $D\left(x_{j}\right)$ from scratch. In contrast, consider that any of the four more sophisticated algorithms will return true in case $L a s t P C_{x_{i}, a_{i}, x_{j}}$ is valid. However,

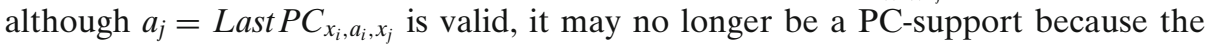
PC-witness for the pair $\left(a_{i}, a_{j}\right)$ in some third variable may have been deleted, and it may be the last one. In a case where $a_{j}$ was the last PC-support in $x_{j}$ for value $a_{i}$, the four advanced algorithms will not delete $a_{i}$ while the brute-force one will. This is because it will exhaustively check all values of $x_{j}$ for PC-support, concluding that there is none.

The worst-case time and space complexities of algorithm 1 maxRPC2 are the same as maxRPC2 . Algorithm 1 maxRPC ${ }^{r m}$ has $\mathrm{O}\left(e n d^{4}\right)$ time and $\mathrm{O}(e d)$ space complexities, which are lower than those of maxRPC ${ }^{r m}$. Experiments with random problems using algorithms 1 maxRPC ${ }^{r m}$ and maxRPC ${ }^{r m}$ showed that the pruning power of lmaxRPC is only slightly weaker than that of maxRPC [28]. At the same time, it can offer significant gains in run times when used during search. These results were also verified by us through a series of experiments on various problem classes.

\subsection{Correctness and complexities}

We now prove the correctness of algorithms maxRPC 3 and maxRPC $3^{r m}$ and analyze their worst-case time and space complexities.

\section{Proposition 1 Algorithm maxRPC3 is sound and complete.}

Proof (Soundness) To prove the soundness of maxRPC3 we must prove that any value that is deleted by maxRPC3 is not maxRPC. Let $a_{i} \in D\left(x_{i}\right)$ be a value that is deleted by maxRPC 3. It is either removed from $D\left(x_{i}\right)$ during the initialization phase (line 14 Function 2) or in line 8 of Algorithm 1, after checkPCsupLoss has returned false, or in line 12, after checkPCsupLoss has returned true and checkPCwitLoss has returned false. 
In the first case, since function initilization checks all values in a brute-force manner, it is clear that any deleted value $a_{i}$ either has no AC-support or none of its AC-supports is a PC-support in some variable $x_{j}$. The non-existence of a PC-support is determined using function searchPCwit whose correctness is discussed below.

In the second case, since checkPCsupLoss returns false, as long as Last $P C_{x_{i}, a_{i}, x_{j}}$ is not valid in Algorithm 1, a new PC-support in $D\left(x_{j}\right)$ is sought (lines 5-11 in Function 3). This search starts with the value at $\max \left(\operatorname{Last}_{P} C_{x_{i}, a_{i}, x_{j}}+1, \operatorname{Last} A C_{x_{i}, a_{i}, x_{j}}\right)$ or at $\max \left(\operatorname{Last}_{P} C_{x_{i}, a_{i}, x_{j}}+1\right.$, Last $\left.A C_{x_{i}, a_{i}, x_{j}}+1\right)$, depending on whether Last $A C_{x_{i}, a_{i}, x_{j}}$ is valid or not. This is correct since any value before $\operatorname{Last} P C_{x_{i}, a_{i}, x_{j}}+1$ and any value before Last $A C_{x_{i}, a_{i}, x_{j}}$ is definitely not an AC-support for $a_{i}$ (similarly for the other case). checkPCsupLoss will return false either because no AC-support for $a_{i}$ can be found in $D\left(x_{j}\right)$, or because for any AC-support found, searchPCwit returned false. In the former case there is no PC-support for $a_{i}$ in $D\left(x_{j}\right)$ since there is no AC-support. In the latter case, for any AC-support $a_{j}$ found there must be some third variable $x_{k}$ for which no PC-witness for the pair $\left(a_{i}, a_{j}\right)$ exists. For each third variable $x_{k}$ searchPCwit correctly identifies a PC-witness if one of the conditions in line 3 holds. In none holds then searchPCwit searches for a PC-witness starting from $\max \left(\operatorname{Last} A C_{x_{i}, a_{i}, x_{k}}\right.$, Last $\left.A C_{x_{j}, a_{j}, x_{k}}\right)$. This is correct since Last $A C_{x_{i}, a_{i}, x_{k}}$ and Last $A C_{x_{j}, a_{j}, x_{k}}$ are updated with the lexicographically smallest support of $a_{i}$ (resp. $\left.a_{j}\right)$ in $D\left(x_{k}\right)$ by calling function searchACsup, meaning that any value smaller than $\max \left(\right.$ Last $A C_{x_{i}, a_{i}, x_{k}}$, Last $\left.A C_{x_{j}, a_{j}, x_{k}}\right)$ is incompatible with either $a_{i}$ or $a_{j}$. Therefore, if searchPCwit returns false then there is no PC-witness for some third variable $x_{k}$. Hence, if checkPCsupLoss returns false, it means no PC-support for $a_{i}$ can be found in $D\left(x_{j}\right)$ and it is thus correctly deleted.

Now assume that $\operatorname{Last} P C_{x_{i}, a_{i}, x_{j}}$ is valid in Algorithm 1 and $a_{i}$ was removed after checkPCwitLoss returned false. This means that for some variable $x_{k}$, constrained with both $x_{i}$ and $x_{j}$, both the first part (lines 3-11) and the second part (line 12) in Function 6 of checkPCwitLoss failed to set the boolean witness to true. Regarding the first part, the failure means that the pair of values $\left(a_{i}, a_{k}\right)$, where $a_{k}$ is the last PC-support of $a_{i}$ in $D\left(x_{k}\right)$ found, has no PC-witness in $D\left(x_{j}\right)$. In more detail, the search for a PC-witness correctly starts from $\max \left(\operatorname{Last} A C_{x_{i}, a_{i}, x_{j}}\right.$, Last $\left.A C_{x_{j}, a_{j}, x_{j}}\right)$, after both Last $A C$ pointers have been updated by searchACsup. The condition in line 4 is similar to the corresponding condition in searchPCwit and thus, if it is true, the search for PC-witness is correctly overriden. Regarding the second part, the failure means that no alternative PC-support for $a_{i}$ in $D\left(x_{k}\right)$ was found. In more detail when calling checkPCsupLoss $\left(a_{i}, x_{k}\right)$, the search for a PC-support starts from $\max \left(\operatorname{Last}_{P} C_{x_{i}, a_{i}, x_{k}}+1\right.$, Last $\left.A C_{x_{i}, a_{i}, x_{k}}\right)$ or $\max \left(\operatorname{Last} P C_{x_{i}, a_{i}, x_{k}}+1\right.$, Last $\left.A C_{x_{i}, a_{i}, x_{k}}+1\right)$, depending on the existence of $\operatorname{Last} A C_{x_{i}, a_{i}, x_{k}}$. This is correct since no earlier value can be a PC-support. If there is no consistent $\left(a_{i}, a_{k}\right)$ pair or searchPCwit returns false for all consistent pairs found, then $a_{i}$ has no PC-support in $D\left(x_{k}\right)$ and is thus correctly deleted.

Completeness To prove the completeness of maxRPC3 we need to show that if a value is not maxRPC then the algorithm will delete it. The initialization function checks all values of all variables one by one in a brute-force manner and removes any value that is not maxRPC. Values that are maxRPC have their Last PC pointers set to the discovered PC-supports. Thereafter, the effects of such removals are propagated by calling Algorithm 1 and as a result new value deletions may occur. 
Now consider a value $a_{i} \in D\left(x_{i}\right)$ that was not removed by the initialization function but after propagation is no longer maxRPC. This is either because of PC-support or PC-witness loss.

In the first case assume that $x_{j}$ is the variable in which $a_{i}$ no longer has a PCsupport. Since the previously found PC-support of $a_{i}$ has been deleted, $x_{j}$ must have been added to $Q$ at some point. When $x_{j}$ is removed from $Q$ all neighboring variables, including $x_{i}$ will be checked. Since Last $P C_{x_{i}, a_{i}, x_{j}}$ is no longer valid function checkPCsupLoss will be called to search for a new PC-support concluding that there is none. Therefore, it will return false and $a_{i}$ will be deleted.

In the second case assume that the pair of values $\left(a_{i}, a_{j}\right)$, where $a_{j}$ is the last PCsupport of $a_{i}$ in $D\left(x_{j}\right)$, has lost its last PC-witness $a_{k}$ in variable $x_{k}$. If $\operatorname{Last} P C_{x_{i}, a_{i}, x_{j}}$ is not valid, which means that $x_{j}$ was added to $Q$, then we have the same case as above. Therefore, after $x_{j}$ is removed from $Q$, checkPCsupLoss will find out that there is no PC-support for $a_{i}$ in $D\left(x_{j}\right)$ and will delete it. If $\operatorname{Last} P C_{x_{i}, a_{i}, x_{j}}$ is valid then checkPCsupLoss will be omitted (line 7 of Algorithm 1). Since $a_{k}$ was deleted, $x_{k}$ was added to $Q$ at some point. When $x_{k}$ is removed from $Q$ all neighboring variables, including $x_{i}$ will be checked. If $a_{i}$ has no longer a PC-support in $D\left(x_{k}\right)$, this will be detected by checkPCsupLoss and $a_{i}$ will be deleted. Otherwise, function checkPCwitLoss will be called. The for loop in line 1 will go through every variable constrained with both $x_{i}$ and $x_{k}$, including $x_{j}$. Since Last $P C_{x_{i}, a_{i}, x_{j}}$ is valid, a new PCwitness for $\left(a_{i}, a_{j}\right)$ in $D\left(x_{k}\right)$ will be sought (lines 3-11). Since $a_{k}$ was the last PCwitness, none will be found and as a result a new PC-support for $a_{i}$ in $D\left(x_{j}\right)$ will be sought (line 12). Since $a_{j}$ was the last PC-support for $a_{i}$ in $D\left(x_{j}\right)$, none will be found, checkPCwitLoss will return false, and $a_{i}$ will be deleted.

\section{Proposition 2 Algorithm maxRPC $3^{\text {rm }}$ is sound and complete.}

Proof The proof is very similar to the corresponding proof for maxRPC3. As explained, the main difference between the two algorithms concerns the use of the Last AC and LastPC structures. As maxRPC $3^{r m}$ does not maintain these structures incrementally, the searches for PC-supports in checkPCsupLoss ${ }^{\text {rm }}$ and checkPCwitLoss ${ }^{r m}$ and the searches for PC-witnesses in searchPCwit ${ }^{r m}$ and checkPCwitLoss $^{r m}$ start from scratch. Clearly, this has no effect on the soundness or completeness of the algorithm since it guarantees that all potential PC-supports and PC-witnesses are checked. Furthermore, the conditions for avoiding redundant searches using residues are the same as in maxRPC3. Finally, another difference between the two algorithms is the exploitation of bidirectionality by maxRPC $3^{r m}$. By the definition of path and arc consistency, bidirectionality holds. That is, when a PC-support (AC-support) $a_{j} \in D\left(x_{j}\right)$ is located for a value $a_{i} \in D\left(x_{i}\right)$ then $a_{i}$ is a PCsupport (AC-support) for $a_{j}$. Since the property of bidirectionality is exploited only to update residues, it does not affect the correctness of the algorithm.

We now discuss the complexities of algorithms maxRPC 3 and maxRPC $3^{r m}$ and their light versions. To directly compare with existing algorithms for (1)maxRPC, the time complexities give the asymptotic number of constraint checks. ${ }^{2}$ Following [23],

\footnotetext{
${ }^{2}$ However, constraint checks do not always reflect run times as other operations may have an equal or even greater effect.
} 
the node time (resp. space) complexity of a (1)maxRPC algorithm is the worst-case time (resp. space) complexity of invoking the algorithm after a decision has been made (e.g. a variable assignment or a value removal). The corresponding branch complexities of an (1)maxRPC algorithm are the worst-case complexities of any incremental sequence of $k \leq n$ invocations of the algorithm. That is, the complexities of incrementally running the algorithm down a branch of the search tree until a fail occurs.

Proposition 3 The node and branch time complexity of (1) maxRPC3 is $O\left(\right.$ end $\left.^{3}\right)$.

Proof The complexity is determined by the total number of calls to function isConsistent in checkPCsupLoss, checkPCwitLoss, and mainly searchPCwit where most checks are executed.

Each variable can be inserted and extracted from $Q$ every time a value is deleted from its domain, giving $\mathrm{O}(d)$ times in the worst case. Each time a variable $x_{j}$ is extracted from $Q$, checkPCsupLoss will look for a PC-support in $D\left(x_{j}\right)$ for all values $a_{i} \in D\left(x_{i}\right)$, s.t. $c_{i, j} \in C$. For each variable $x_{i}, \mathrm{O}(d)$ values are checked. Checking if a value $a_{j} \in D\left(x_{j}\right)$ is a PC-support involves first checking in $\mathrm{O}(1)$ if it is an ACsupport (line 6 in checkPCsupLoss) and then calling searchPCwit (line 9). The cost of searchPCwit is $\mathrm{O}(n+n d)$ since there are $\mathrm{O}(n)$ variables constrained with both $x_{i}$ and $x_{j}$ and, after making the checks in line 3, their domains must be searched for a $\mathrm{PC}$-witness, each time from scratch with cost $\mathrm{O}(n d)$. Through the use of Last PC no value of $x_{j}$ will be checked more than once over all the $\mathrm{O}(d)$ times $x_{j}$ is extracted from $Q$, meaning that for any value $a_{i} \in D\left(x_{i}\right)$ and any variable $x_{j}$, the overall cost of searchPCwit will be $\mathrm{O}\left(d n+n d^{2}\right)=\mathrm{O}\left(n d^{2}\right)$. Hence, checkPCsupLoss will cost $\mathrm{O}\left(n d^{2}\right)$ for one value of $x_{i}$, giving $\mathrm{O}\left(n d^{3}\right)$ for $d$ values. Since, in the worst case, this process will be repeated for every pair of variables $x_{i}$ and $x_{j}$ that are constrained, the total cost of checkPCsupLoss will be $\mathrm{O}\left(e n d^{3}\right)$. This is the node complexity of ImaxRPC3.

In checkPCwitLoss the algorithm iterates over the variables in a triangle with $x_{j}$ and $x_{i}$. In the worst case, for each such variable $x_{k}, D\left(x_{j}\right)$ will be searched from scratch for a PC-witness of $a_{i}$ and its current PC-support in $x_{k}$. As $x_{j}$ can be extracted from $Q \mathrm{O}(d)$ times and each search from scratch costs $\mathrm{O}(d)$, the total cost of checking for a PC-witness in $D\left(x_{j}\right)$, including the checks of line 4 in checkPCwitLoss, will be $\mathrm{O}\left(d+d^{2}\right)$. For $d$ values of $x_{i}$ this will be $\mathrm{O}\left(d^{3}\right)$. As this process will be repeated for all triangles of variables, whose number is bounded by en, its total cost will be $\mathrm{O}\left(e n d^{3}\right)$. If no PC-witness is found then a new PC-support for $a_{i}$ in $D\left(x_{k}\right)$ is sought through searchPCwit. This costs $\mathrm{O}\left(n d^{2}\right)$ as explained above but it is amortized with the cost incurred by the calls to searchPCwit from checkPCsupLoss. Therefore, the cost of checkPCwitLoss is $\mathrm{O}\left(e n d^{3}\right)$. This is also the node complexity of $\operatorname{maxRPC} 3$.

The branch complexity of $(1) \operatorname{maxRPC} 3$ is also $\mathrm{O}\left(e n d^{3}\right)$. This is because the use of Last $P C$ ensures that for any constraint $c_{i, j}$ and a value $a_{i} \in D\left(x_{i}\right)$, each value of $x_{j}$ will be checked at most once for PC-support while going down the branch. Therefore, the cost of searchPCwit is amortized.

Proposition 4 The node and branch time complexities of 1 maxRPC $3^{\text {rm }}$ and maxRPC $3^{r m}$ are $O\left(e n d^{4}\right)$ and $O\left(e n^{2} d^{4}\right)$ respectively. 
Proof The proof is similar to that of Proposition 3. The main difference with ImaxRPC 3 is that since last PC is not updated incrementally, each time we seek a PCsupport for a value $a_{i} \in D\left(x_{i}\right)$ in $x_{j}, D\left(x_{j}\right)$ will be searched from scratch in the worst case. This incurs an extra $\mathrm{O}(d)$ cost to checkPCsupLoss ${ }^{r m}$ and searchPCwit ${ }^{r m}$. Hence, the node complexity of ImaxRPC $3^{r m}$ is $\mathrm{O}\left(\right.$ end $\left.^{4}\right)$. Also, the total cost of searchPCwit ${ }^{r m}$ in one node cannot be amortized. This means that the cost of searchPCwit ${ }^{r m}$ when called within checkPCwitLoss ${ }^{r m}$ is $\mathrm{O}\left(n d^{2}\right)$. Hence, the node complexity of maxRPC $3^{r m}$ is $\mathrm{O}\left(e n^{2} d^{4}\right)$. The branch complexities are the same because the calls to searchPCwit ${ }^{r m}$ are amortized.

The space complexities of the algorithms are determined by the space required for data structures Last $P C$ and Last $A C$. Since both require $\mathrm{O}(e d)$ space, this is the node space complexity of (1) maxRPC3 and (1) maxRPC $3^{r m}$. (l) maxRPC3 has $\mathrm{O}($ end $)$ branch space complexity because of the extra space required for the incremental update and restoration of the data structures. As (1) maxRPC $3^{r m}$ avoids this, its branch space complexity is $\mathrm{O}(e d)$.

\section{Further exploitation of residues in maxRPC algorithms}

As detailed above, the use of the Last $P C$ and Last $A C$ data structures by algorithms such as maxRPC2, maxRPC3, and AC2001/3.1 can give optimal time complexity bounds. However, the overhead for maintaining the required data structures during search can outweigh the benefit of the optimal theoretical results. On the other hand, the use the Last $P C$ and Last $A C$ structures as residues by algorithms such as maxRPC ${ }^{r m}$, maxRPC $3^{r m}$, and $A C 3^{r m}$ sacrifices the optimal time complexity to achieve better average performance in practice. ${ }^{3}$

In this section we investigate variants of $\operatorname{maxRPC} 3^{r m}$ that offer a compromise between maxRPC $3^{r m}$ and maxRPC 3 by exploring ideas presented in [23] regarding the use of residues in $\mathrm{AC}$ algorithms. The first variant of maxRPC3 ${ }^{r m}$, called maxRPC3-resopt, uses an extra data structure to record the current PC-supports before the invocation of the maxRPC algorithm at each node of the search tree. As explained below, by exploiting this data structure we can achieve an improved node time complexity. The second variant, called maxRPC3-start, also introduces an additional data structure, but only makes use of information obtained during the initialization phase of the maxRPC algorithm. This does not improve the asymptotic time complexity, but results in better average performance in practice.

\section{1 maxRPC3-resOpt}

Algorithm maxRPC3-resOpt is inspired from the ACS-resOpt algorithm of [23]. Adapting the main idea of ACS-resOpt to maxRPC, we use a data structure, called Stop, to copy and remember the residues in Last PC each time a node is visited right before the maxRPC algorithm is invoked. Also, we view each domain as being "circular". That is, the last value in the initial domain of a variable is followed

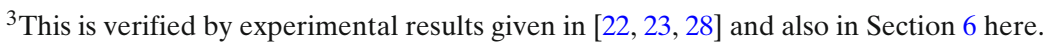




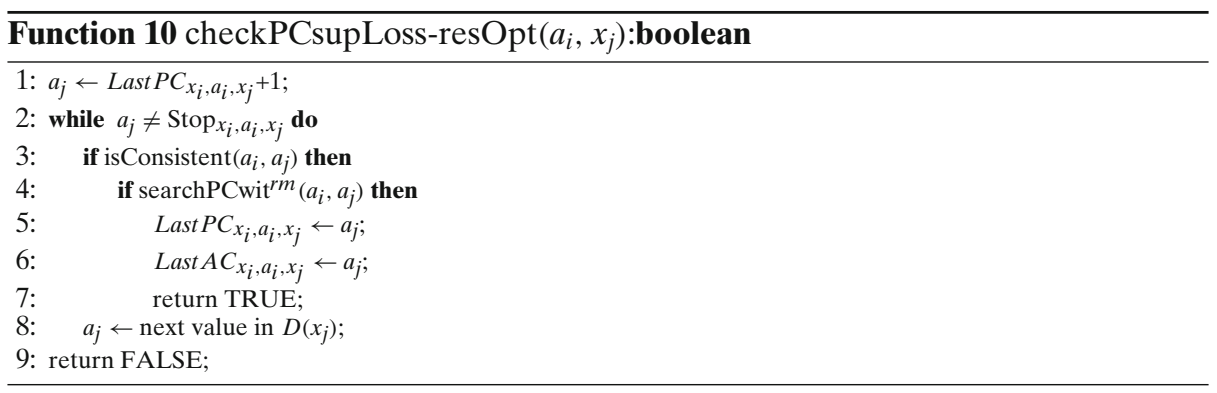

by the first value. Once a branching decision is made (e.g. variable assignment), maxRPC3-res Opt copies all the Last PC residues to the Stop data structure. Then, as maxRPC3-resOpt is executed at this specific node, the search for a new PCsupport for $a_{i} \in D\left(x_{i}\right)$ in $D\left(x_{j}\right)$ starts from the value immediately after $\operatorname{Last} P C_{x_{i}, a_{i}, x_{j}}$, continues through the end of the domain, if no PC-support is found, and back to the start of the domain until it encounters Stop $_{x_{i}, a_{i}, x_{j}}$. This may save many checks since, unlike maxRPC $3^{r m}$, each value in $D\left(x_{j}\right)$ can be checked for PC-support at most once.

We now explain in detail functions checkPCsupLoss-resOpt and checkPCwitLossresOpt, that replace functions checkPCsupLoss ${ }^{r m}$ and checkPCwitLoss ${ }^{r m}$. In function checkPCsupLoss-resOpt (Function 10), we set $a_{j}$ to the next value after Last $P C_{x_{i}, a_{i}, x_{j}}$, which is the first value to be checked for being a $\mathrm{PC}$-support in line 1 . When the search for PC-support encounters Stop $_{x_{i}, a_{i}, x_{j}}$ (line 2), all possible PC-supports will have been examined. Note that since we consider the domains to be circular, once the last available value in $D\left(x_{j}\right)$ has been unsuccessfuly checked, the search for PCsupport will continue from the start of $D\left(x_{j}\right)$. That is, in line $8 a_{j}$ will be set to the first available value in $D\left(x_{j}\right)$.

A significant difference from maxRPC $3^{r m}$ is that maxRPC3-resopt cannot exploit the bidirectionality of LastPC. When a PC-support $a_{j} \in D\left(x_{j}\right)$ is found for $a_{i} \in D\left(x_{i}\right)$ then only $\operatorname{Last} P C_{x_{i}, a_{i}, x_{j}}$ is set to $a_{j}$. We do not set $\operatorname{Last} P C_{x_{j}, a_{j}, x_{i}}$ to $a_{i}$, as done in maxRPC $3^{r m}$, because bidirectionality no longer holds. To demonstrate this, assume that during the application of maxRPC3-resOpt at some node, we discover the PC-support $a_{j} \in D\left(x_{j}\right)$ for $a_{i} \in D\left(x_{i}\right)$ and through bidirectionality Last $P C_{x_{j}, a_{j}, x_{i}}$ is set to $a_{i}$. Now a later point in search when maxRPC $3^{r m}$ is invoked we set Stop $x_{j}, a_{j}, x_{i}$ to $a_{i}$ and continue propagation. If during the search for PC-support for value $a_{i}^{\prime} \in D\left(x_{i}\right)$, $a_{i}^{\prime} \neq a_{i}$, in $D\left(x_{j}\right)$ we discover $a_{j}$ then Last $P C_{x_{j}, a_{j}, x_{i}}$ will be set to $a_{i}^{\prime}$. Now assume that later we seek a PC-support for $a_{j}$ in $D\left(x_{i}\right)$ and $a_{i}^{\prime}$ is no longer valid. Then all values located between $a_{i}$ and $a_{i}^{\prime}$ will be skipped because the search will start at $a_{i}^{\prime}+1$ and will terminate when $a_{i}=$ Stop $p_{x_{j}, a_{j}, x_{i}}$ is reached. Consequently, bidirectionality cannot be exploited. To this end, the auxilary variable $\mathrm{rm}$, used in initialization function is set to false to skip line 11.

On the other hand, Last $A C$ is used as in maxRPC $3^{r m}$ and thus it is updated when a PC-support is found, since this is also an AC-support. Furthermore, the search for a PC-witness for a pair of values is conducted by searchPCwit ${ }^{r m}$, as the changes concern Last $P C$ and do not affect Last $A C$.

Function checkPCwitLoss-resOpt is called when $a_{j}$ is not removed by checkPCsupLoss-resOpt. The pseudocode is simply described in textual form, since it is the same as in checkPCwitLoss ${ }^{r m}$ (Function 9) until line 11. The second part of 
the function (line 12) is executed when Last $P C_{x_{i}, a_{i}, x_{k}}$ is not valid (line 3), or because there is no PC-witness for the pair $\left(a_{i}, a_{k}\right)$ in $D\left(x_{j}\right)$. In these cases a new PC-support for $a_{i}$ is sought in $D\left(x_{k}\right)$, and this is done essentially in the same way as in function checkPCsupLoss-resOpt (Function 10).

Comparing with previous algorithms, maxRPC3-resOpt is sound and complete, as no supports nor witnesses can be overlooked and thus the proof of correctness is very similar to the one given for maxRPC $3^{r m}$. The node time complexity of (1) maxRPC3-resopt is $\mathrm{O}\left(\right.$ end $\left.^{3}\right)$, the same as maxRPC3, since the search for a new PC-support starts from Last $P C+1$ and not from scratch as in maxRPC $3^{r m}$. Before maxRPC3-resOpt is invoked, we setStop $=$ Last $P C$ and thus for any constraint $c_{i, j}$ and a value $a_{i} \in D\left(x_{i}\right)$, each value of $x_{j}$ will be checked at most $n d$ times for PCsupport while going down the branch. As a result the branch complexity is $\mathrm{O}\left(e n^{2} d^{4}\right)$. The node space complexity is determined by the space required for storing the Last AC, Last PC, and Stop structures, which is $\mathrm{O}(e d)$. The branch space complexity is also $\mathrm{O}(e d)$, because the data structures are not copied/restored.

Although maxRPC3-resOpt achieves a better node complexity than maxRPC ${ }^{r m}$, it carries the additional overhead of having to initialize the Stop data structure at each node of the search tree. Experiments in Section 6 show that this is indeed an important drawback. The copying of Last PC to Stop at each node (in $O(e d)$ time) results in higher cpu times, despite the savings in constraint checks.

\section{2 maxRPC3-start}

A simple way to reduce the number of constraint checks, when a value in LastPC is not valid, is to keep track of the first PC-support found after preprocessing. The version of maxRPC $3^{r m}$ presented here, called maxRPC3-start, stores this value in a structure we call LeftMostPC, with $O(e d)$ size. In case of PC-support loss, instead of searching from scratch, we start from the value stored in LeftMost PC that contains the first PC-support found in the initialization function. Thus, we omit values between the first value in a domain and the LeftMost $P C$ value to save redundant checks. For every value $a_{i} \in D\left(x_{i}\right)$ and constraint $c_{i, j}$, LeftMost $P C_{x_{i}, a_{i}, x_{j}}$ is initialized to NIL, like the Last PC and Last AC structures, and it is updated in the initialization function, exactly when Last $P C$ is updated. To obtain algorithm maxRPC3-start from maxRPC $3^{r m}$, we make the following simple changes.

- We insert in line 11 of initialization the assignment:

$$
\text { LeftMost } P C_{x_{i}, a_{i}, x_{j}} \leftarrow a_{j} \text {; }
$$

Note that while we still exploit the bidirectionality of Last $P C$, this property does not hold for LeftMost PC. That is, if the first PC-support for $a_{i}$ in $D\left(x_{j}\right)$ is value $a_{j}$, this does not necessarily mean that the first PC-support for $a_{j}$ in $D\left(x_{i}\right)$ is $a_{i}$.

- In order to start the search for a new PC-support from the first PC-support found, we replace line 1 in checkPCsupLoss ${ }^{\text {rm }}$ with:

$$
\text { 1: for each } a_{j} \in D\left(x_{j}\right), a_{j} \geq \operatorname{LeftMost} P C_{x_{i}, a_{i}, x_{j}} \text { do }
$$

This change will affect also the checkPCwitLoss ${ }^{r m}$ function which calls checkPCsupLoss ${ }^{\text {rm }}$ in line 12. 
Table 1 Time and space complexities of (1)maxRPC algorithms

\begin{tabular}{llll}
\hline Algorithm & $\begin{array}{l}\text { Time } \\
\text { complexity }\end{array}$ & $\begin{array}{l}\text { Space } \\
\text { complexity }\end{array}$ & $\begin{array}{l}\text { Maintains } \\
\text { structures }\end{array}$ \\
\hline maxRPC1 & $\mathrm{O}\left(e n d^{3}\right)$ & $\mathrm{O}(e n d)$ & Yes \\
maxRPC2 & $\mathrm{O}\left(e n d^{3}\right)$ & $\mathrm{O}(e n d)$ & Yes \\
maxRPC3 & $\mathrm{O}\left(e n d^{3}\right)$ & $\mathrm{O}(e n d)$ & Yes \\
maxRPC ${ }^{r m}$ & $\mathrm{O}\left(e n^{2} d^{4}\right)$ & $\mathrm{O}(e n d)$ & No \\
maxRPC3 & $\mathrm{O}\left(e n^{2} d^{4}\right)$ & $\mathrm{O}(e d)$ & No \\
maxRPC3-resOpt & $\mathrm{O}\left(e n^{2} d^{4}\right)$ & $\mathrm{O}(e d)$ & No \\
maxRPC3-start & $\mathrm{O}\left(e n^{2} d^{4}\right)$ & $\mathrm{O}(e d)$ & No \\
lmaxRPC2 & $\mathrm{O}\left(e n d^{3}\right)$ & $\mathrm{O}(e n d)$ & Yes \\
lmaxRPC3 & $\mathrm{O}\left(e n d^{3}\right)$ & $\mathrm{O}(e n d)$ & Yes \\
lmaxRPC ${ }^{r m}$ & $\mathrm{O}\left(e n d^{4}\right)$ & $\mathrm{O}(e d)$ & No \\
lmaxRPC3 & $\mathrm{O}\left(e n d^{4}\right)$ & $\mathrm{O}(e d)$ & No \\
lmaxRPC3-resOpt & $\mathrm{O}\left(e n d^{4}\right)$ & $\mathrm{O}(e d)$ & No \\
lmaxRPC3-start & $\mathrm{O}\left(e n d^{4}\right)$ & $\mathrm{O}(e d)$ & No \\
\hline
\end{tabular}

maxRPC3-start is sound and complete as it is guaranteed than no value earlier than the corresponding LeftMost PC value can be a potential PC-support for some value $a_{i} \in D\left(x_{i}\right)$. The node and branch time complexity of maxRPC3-start is $O\left(e n^{2} d^{4}\right)$, the same as maxRPC $3^{r m}$, as in the worst case, the LeftMostPC values are the first values in each variable's domain. ImaxRPC3-start is the light version that results from removing the corresponding checkPCwitLoss-start function. Its complexity is $O\left(e n d^{4}\right)$, the same as 1 maxRPC $3^{r m}$.

Table 1 summarises the asymptotic branch time and space complexities of the available (1)maxRPC algorithms. Under the column "maintains structures" we indicate whether a given algorithm requires to incrementally maintain some data structure or not.

\section{Heuristics for maxRPC algorithms}

Numerous heuristics for ordering constraint or variable revisions have been proposed and used within AC algorithms [2, 11, 17, 29]. Generally, many constraint solvers employ heuristics to order the application of propagators or/and the revision of variables and constraints [27]. Heuristics such as the ones used by AC algorithms can be also used within a maxRPC algorithm to efficiently select the next variable to be removed from the propagation list. In addition to this, maxRPC and lmaxRPC algorithms can benefit from the use of heuristics elsewhere in their execution. Once a variable $x_{j}$ has been removed from the propagation list, heuristics can be applied in many ways in either a maxRPC or a lmaxRPC algorithm. In the following we summarize the possibilities of heuristic using algorithm ( I) maxRPC3 for illustration.

H1 A heuristic can be used to select the next variable $x_{j}$ to remove from the propagation list $Q$ (line 4 of Algorithm 1). Such heuristics are successfully used within AC algorithms.

H2 After a variable $x_{j}$ is removed from $Q$ all neighboring variables $x_{i}$ are revised. lmaxRPC (resp. maxRPC) will detect a failure if the condition of PC-support loss (resp. either PC-support or PC-witness loss) occurs for all values of $x_{i}$. In 
such situations, the sooner $x_{i}$ is considered and the failure is detected, the more constraint checks will be saved. Hence, the order in which the neighboring variables of $x_{j}$ are considered can be determined using a fail-first type of heuristic (line 5 of Algorithm 1).

H3 Once an AC-support $a_{j} \in D\left(x_{j}\right)$ has been found for a value $a_{i} \in D\left(x_{i}\right)$, we try to establish if it is a PC-support. If there is no PC-witness for the pair $\left(a_{i}, a_{j}\right)$ in some variable $x_{k}$ then $a_{j}$ is not a PC-support. Therefore, we can again use failfirst heuristics to determine the order in which the variables forming a triangle with $x_{i}$ and $x_{j}$ are considered (line 1 of Function searchPCwit).

The above cases apply to both ImaxRPC and maxRPC algorithms. In addition, a maxRPC algorithm can employ heuristics as follows:

H4 For each value $a_{i} \in D\left(x_{i}\right)$ and each variable $x_{k}$ constrained with both $x_{i}$ and $x_{j}$, Function checkPCwitLoss checks if the pair $\left(a_{i}, a_{k}\right)$ still has a PC-witness in $D\left(x_{j}\right)$. Again heuristics can be used to determine the order in which the variables constrained with $x_{i}$ and $x_{j}$ are considered (line 1 of checkPCwitLoss).

H5 In Function checkPCwitLoss, a new PC-support for $a_{i}$ in $D\left(x_{k}\right)$ may be seeked. The order in which variables constrained with both $x_{i}$ and $x_{k}$ are considered can be determined heuristically as in the case of $\mathrm{H} 3$ above (within the call to searchPCwit).

As explained, the purpose of such ordering heuristics will be to "fail-first" [19]. That is, to quickly discover potential failures (in the case of $\mathrm{H} 2$ above), refute values that are not PC-supports ( $\mathrm{H} 3$ and $\mathrm{H} 5)$ and delete values that have no PC-support (H3). Such heuristics can be applied within any coarse-grained maxRPC algorithm to decide the order in which variables are considered. Examples of heuristics that can be used are the following.

dom Consider the variables in ascending domain size. This heuristic can be applied in any of the five cases.

del_ratio Consider the variables in ascending ratio of the number of remaining values to the initial domain size. This heuristic can be applied in any of the five cases.

wdeg For $\mathrm{H} 1$ consider the variables in descending weighted degree. For H2 consider the variables $x_{i}$ in descending weight for the constraint $c_{i, j}$. In the case of $\mathrm{H} 3$ consider the variables $x_{k}$ in descending average weight for the constraints $c_{i, k}$ and $c_{j, k}$. Similarly for $\mathrm{H} 4$ and $\mathrm{H} 5$.

dom/wdeg Consider the variables in ascending value of dom/wdeg. This heuristic can be applied in any of the five cases.

Experiments demonstrated that applying heuristics $\mathrm{H} 1$ and $\mathrm{H} 2$ can sometimes be effective, while doing so for $\mathrm{H} 3, \mathrm{H} 4$, and $\mathrm{H} 5$ may save constraint checks but usually penalizes cpu times because of the overhead involved in computating the heuristics. Although the primal purpose of the heuristics is to save constraint checks, it is interesting to note that some of the heuristics can also divert search to different areas of the search space when a variable ordering heuristic like dom/wdeg is used, resulting in fewer node visits. For example, two different orderings of the variables in the case of $\mathrm{H} 2$ may result in different constraints causing a failure. As dom/wdeg increases the weight of a constraint each time it causes a failure and uses the weights 
to select the next variable, this may later result in different branching choices. This is explained for the case of $\mathrm{AC}$ in [2].

\section{Experiments}

To evaluate the various maxRPC algorithms, we experimented with several classes of structured and random binary CSPs taken from C.Lecoutre's XCSP repository. Excluding instances that were very hard for all algorithms, our evaluation was done on 200 instances in total from various problem classes (see Table 2). More details about these instances can be found in C.Lecoutre's homepage. ${ }^{4}$

All algorithms used the dom/wdeg heuristic for variable ordering [12] and lexicographic value ordering. As explained in Section 2, dom/wdeg increases the weight of a constraint when this constraint causes a value removal. This process is rather straightforward when AC is used for constraint propagation, but perhaps not so when stronger local consistencies are used. For the case of maxRPC we chose to increase constraint weights in the following way. When a failure occurs, the weight of constraint $c_{i, j}$ is updated, right after line 7 and 11 of Algorithm 1 and after line 13 in the initialization function.

In all following tables, the results of the best algorithm, with respect to run-time, are highlighted with bold. If not explicitly mentioned, the propagation list $Q$ was implemented as a FIFO queue and no heuristic from Section 5 was used.

Table 2 compares the performance of stand-alone algorithms used for preprocessing. We give average results for all the instances, grouped into specific problem classes. We include results from coarse-grained maxRPC algorithms, maxRPC2, $\operatorname{maxRPC} 3, \operatorname{maxRPC}^{r m}$, maxRPC $3^{r m}$ and from their corresponding light versions.

Regarding existing algorithms, results demonstrate that maxRPC ${ }^{r m}$ is particularly costly on large instances because of the penalties associated in initializing its data structures. Specifically, this algorithm timed out on some large instances of the Queens problem class, which explains the empty data entries in the table. In comparison, maxRPC2 displays a better average performance which is not surprising given its lower complexity. The new algorithm maxRPC3 is very close to maxRPC2 in run times, apart from the first and last classes where it is notably faster. The same holds for maxRPC $3^{r m}$ with the exception of the geometric class where it is clearly worse than the rest of the algorithms. Any gain in performance displayed by the new algorithms is due to the elimination of many redundant constraint checks as the corresponding numbers show.

Comparing light to full maxRPC algorithms it is perhaps surprising that the light versions typically achieve the same number of value deletions as their full counterparts. This means that approximation algorithms for maxRPC are quite effective. Any differences in value deletions among maxRPC algorithms are caused by the different order of operations in which inconsistency is discovered for some instances. In classes where the constraints checks for a maxRPC and a corresponding lmaxRPC algorithm are the same or very close, there are very few, if any, value deletions.

$\overline{{ }^{4} \text { http://www.cril.univ-artois.fr/ lecoutre/benchmarks.html }}$ 


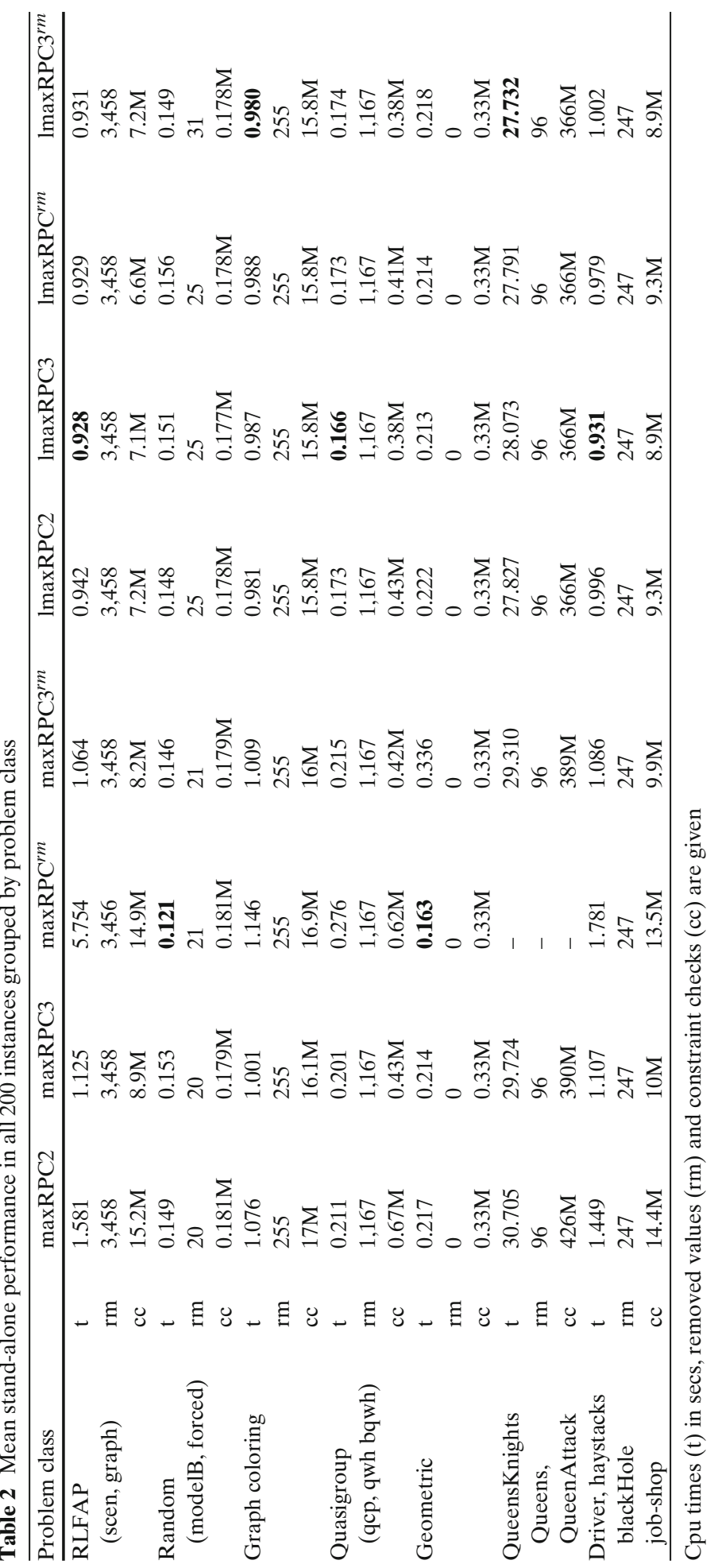


Table 3 compares the performance of search algorithms that apply lmaxRPC throughout search on several problem classes including instances from RLFAPs, random, Quasigroup, geometric, and Queen problems. These instances have been selected to demonstrate cases where either the new algorithms achieve a clear improvement making the best algorithm among them outperform or compete with MAC, or cases where, despite the improvement, the maxRPC-based algorithms are still significantly inferior to MAC. Hence, we present some extreme behaviors for both situations. The algorithms compared are $1 \mathrm{maxRPC}{ }^{r m}, 1 \mathrm{maxRPC} 3^{r m}$, lmaxRPC3-resOpt and ImaxRPC3-start. We do not present results from

Table 3 Cpu times ( $\mathrm{t}$ ) in secs, nodes (n) and constraint checks (cc) from various instances

\begin{tabular}{|c|c|c|c|c|c|c|}
\hline Instance & & $\mathrm{AC} 3^{r m}$ & $\operatorname{lmaxRPC}^{r m}$ & $\operatorname{lmaxRPC}^{\mathrm{rm}}$ & lmaxRPC3-resOpt & lmaxRPC3-start \\
\hline \multirow[t]{3}{*}{ scen11-f7 } & $\mathrm{t}$ & 109.3 & 482.6 & 186.6 & 214 & 159.4 \\
\hline & $\mathrm{n}$ & 353,901 & 76,954 & 76,954 & 57,037 & 76,954 \\
\hline & $\mathrm{cc}$ & $467 \mathrm{M}$ & $5,184 \mathrm{M}$ & $1,596 \mathrm{M}$ & $1,011 \mathrm{M}$ & $1,323 \mathrm{M}$ \\
\hline \multirow[t]{3}{*}{ graph9-f9 } & $\mathrm{t}$ & 8.7 & 54.5 & 21 & 45.6 & 17.5 \\
\hline & $\mathrm{n}$ & 46,705 & 16,839 & 16,839 & 14,838 & 16,839 \\
\hline & $\mathrm{cc}$ & $25 \mathrm{M}$ & $458 \mathrm{M}$ & $184 \mathrm{M}$ & $145 \mathrm{M}$ & $153 \mathrm{M}$ \\
\hline \multirow{3}{*}{$\begin{array}{c}\text { rand-2-40-11- } \\
414-200-30\end{array}$} & $\mathrm{t}$ & 14.1 & 17.6 & 11.6 & 8.9 & 11.5 \\
\hline & $\mathrm{n}$ & 164,958 & 28,655 & 28,655 & 21,105 & 28,655 \\
\hline & $\mathrm{cc}$ & $61 \mathrm{M}$ & $249 \mathrm{M}$ & $98 \mathrm{M}$ & $70 \mathrm{M}$ & $97 \mathrm{M}$ \\
\hline \multirow[t]{3}{*}{ will199GPIA-6 } & $\mathrm{t}$ & 1.6 & 5.9 & 2.5 & 7.9 & 2.7 \\
\hline & $\mathrm{n}$ & 6,996 & 3,316 & 3,316 & 4,300 & 3,316 \\
\hline & $\mathrm{cc}$ & $6 \mathrm{M}$ & $53 \mathrm{M}$ & $21 \mathrm{M}$ & $22 \mathrm{M}$ & $20 \mathrm{M}$ \\
\hline \multirow[t]{3}{*}{ qсp150-120-5 } & $\mathrm{t}$ & 22.9 & 29.3 & 15.5 & 73.8 & 15.7 \\
\hline & $\mathrm{n}$ & 525,629 & 130,384 & 130,384 & 237,644 & 130,384 \\
\hline & $\mathrm{cc}$ & $37 \mathrm{M}$ & $265 \mathrm{M}$ & $43 \mathrm{M}$ & $69 \mathrm{M}$ & $42 \mathrm{M}$ \\
\hline \multirow[t]{3}{*}{ qсp150-120-9 } & $\mathrm{t}$ & 95.2 & 120.6 & $\mathbf{5 7 . 7}$ & 157.4 & 59 \\
\hline & $\mathrm{n}$ & $2,437,173$ & 627,679 & 627,679 & 617,662 & 627,679 \\
\hline & $\mathrm{cc}$ & $157 \mathrm{M}$ & $1,060 \mathrm{M}$ & $163 \mathrm{M}$ & $151 \mathrm{M}$ & $162 \mathrm{M}$ \\
\hline \multirow[t]{3}{*}{ qwh20-166-1 } & $\mathrm{t}$ & 15.3 & 21.7 & 12.2 & 42.7 & 12.8 \\
\hline & $\mathrm{n}$ & 234,095 & 54,286 & 54,286 & 31,346 & 54,286 \\
\hline & $\mathrm{cc}$ & $19 \mathrm{M}$ & $156 \mathrm{M}$ & $18 \mathrm{M}$ & $10 \mathrm{M}$ & $18 \mathrm{M}$ \\
\hline \multirow[t]{3}{*}{ qwh20-166-6 } & $\mathrm{t}$ & 758.3 & 462.5 & 245.9 & $3,342.5$ & 256.2 \\
\hline & $\mathrm{n}$ & $10,691,633$ & 984,555 & 984,555 & $2,364,104$ & 984,555 \\
\hline & $\mathrm{cc}$ & $911 \mathrm{M}$ & $3,381 \mathrm{M}$ & $377 \mathrm{M}$ & $921 \mathrm{M}$ & $372 \mathrm{M}$ \\
\hline \multirow[t]{3}{*}{ qwh20-166-7 } & $\mathrm{t}$ & 64.5 & 46.2 & 24.7 & 319.3 & 26.3 \\
\hline & $\mathrm{n}$ & $1,050,144$ & 124,212 & 124,212 & 241,184 & 124,212 \\
\hline & $\mathrm{cc}$ & $85 \mathrm{M}$ & $342 \mathrm{M}$ & $40 \mathrm{M}$ & $75 \mathrm{M}$ & $39 \mathrm{M}$ \\
\hline \multirow[t]{3}{*}{ geo $50-20-d 4-75-1$} & $\mathrm{t}$ & 54.4 & 248.2 & 140.5 & 143.7 & 145.7 \\
\hline & $\mathrm{n}$ & 260,996 & 122,750 & 122,750 & 124,535 & 122,750 \\
\hline & $\mathrm{cc}$ & $6 \mathrm{M}$ & $1,454 \mathrm{M}$ & $377 \mathrm{M}$ & $1,376 \mathrm{M}$ & $375 \mathrm{M}$ \\
\hline \multirow[t]{3}{*}{ queenAttacking6 } & $\mathrm{t}$ & 32.9 & 60.8 & 23.9 & 94 & 24.3 \\
\hline & $\mathrm{n}$ & 234,759 & 18,488 & 18,488 & 137,731 & 18,488 \\
\hline & $\mathrm{cc}$ & $104 \mathrm{M}$ & $888 \mathrm{M}$ & $242 \mathrm{M}$ & $860 \mathrm{M}$ & $238 \mathrm{M}$ \\
\hline \multirow{3}{*}{$\begin{array}{l}\text { queensKnights- } \\
\text { 15-5-mul }\end{array}$} & $\mathrm{t}$ & 3.1 & 27.6 & 16.5 & 13.4 & 11.6 \\
\hline & $\mathrm{n}$ & 5,819 & 3,5862 & 3,586 & 2,924 & 3,586 \\
\hline & $\mathrm{cc}$ & $23 \mathrm{M}$ & $462 \mathrm{M}$ & $233 \mathrm{M}$ & $174 \mathrm{M}$ & $183 \mathrm{M}$ \\
\hline \multirow[t]{3}{*}{ haystacks-05 } & $\mathrm{t}$ & 4.5 & 2.6 & 2 & 2.8 & 1.8 \\
\hline & $\mathrm{n}$ & $1,182,023$ & 167,629 & 167,629 & 223,547 & 167,629 \\
\hline & $\mathrm{cc}$ & $13 \mathrm{M}$ & $13 \mathrm{M}$ & $7 \mathrm{M}$ & $10 \mathrm{M}$ & $6 \mathrm{M}$ \\
\hline
\end{tabular}


$\operatorname{maxRPC}{ }^{r m}$ and maxRPC ${ }^{r m}$, since these two algorithms, and especially maxRPC ${ }^{r m}$, are inferior to the light versions when used during search. To be precise, maxRPC $3^{r m}$ is competitive on some instances but clearly worse on average. On the other hand, $\operatorname{maxRPC} C^{r m}$ is substantially slower on all the tested instances and exceeds the time limit of two hours on the hardest among them. Algorithms (1) maxRPC2 and (1) maxRPC3 are even less competitive when used during search, because of the overheads for the copying and restoration of the LastPC and LastAC data structures. ( I) maxRPC3 is typically more efficient than (1) maxRPC2.

In general, any maxRPC algorithm is clearly inferior to the corresponding light version when applied during search. The reduction in visited nodes achieved by the former is relatively small and does not compensate for the higher run times of enforcing maxRPC. To put the performance of the lmaxRPC algorithms in perspective, we include results from $\mathrm{MAC}^{r m}$ which is considered one of the most efficient versions of MAC [22, 23]. All of the algorithms used a 2-way branching scheme. ${ }^{5}$

Experiments showed that $\operatorname{lmaxRPC} \mathrm{C}^{r m}$ is the most efficient among existing algorithms when applied during search, which confirms the results given in [28]. Accordingly, lmaxRPC $3^{r m}$ is the most efficient among our algorithms. It is over two times faster than lmaxRPC ${ }^{r m}$ on hard instances, while algorithms lmaxRPC3-resOpt and lmaxRPC3-start are also competitive in many instances. The overhead of copying Last PC to Stop causes lmaxRPC3-resOpt to slow down search in many cases, despite the reduction in the number of constraint checks.

Instance qwh20-166-6 is a pathological case for lmaxRPC3-resopt as this algorithm requires considerable effort compared to the other algorithms. Recall that this algorithm does not exploit the bidirectionality of support, as explained in Section 4.1, meaning that variable revisions, constraint checks, and failures may occur in different orders compared to other algorithms. Through the interaction with the dom/wdeg variable ordering heuristic this may cause a different search direction (see discussion at the end of Section 5), explaining the pathological case.

lmaxRPC3-start and lmaxRPC $3^{r m}$ have similar performance when the numbers of constraint checks are similar. More precisely, lmaxRPC3-start is better only when the PC-support found in preprocessing is lexicographically bigger from the first value in any domain. Since this case does not occur very often, there are no significant benefits when compared to $l$ maxRPC $3^{r m}$ that starts searching from scratch.

Importantly, the speed-ups obtained can make a search algorithm that efficiently applies lmaxRPC competitive with MAC on many instances. For instance, in qwh20166-6 ImaxRPC $3^{r m}$ achieves a better run time than MAC by a factor of three while lmaxRPC ${ }^{r m}$ is 2 times slower compared to 1 maxRPC $3^{r m}$.

We can see that our methods can reduce the numbers of constraint checks by as much as one order of magnitude (e.g. in quasigroup problems qcp and qwh). This is mainly due to the elimination of redundant checks inside function searchPCwit. Cpu times are not cut down by as much, but a speed-up of more than 2 times can be obtained (e.g. qcp150-120-9 and qwh20-166-6). However, there are still many instances where MAC remains considerably faster despite the improvements (e.g. graph9-f9, geo50-20-d4-75-1).

${ }^{5}$ The results reported in [1] were obtained using a $d$-way branching scheme. 
Table 4 Mean search performance in all 200 instances grouped by class

\begin{tabular}{|c|c|c|c|c|c|c|}
\hline Problem class & & $\mathrm{AC}^{r m}$ & $\operatorname{lmaxRPC}^{r m}$ & $\operatorname{lmaxRPC} 3^{r m}$ & lmaxRPC3-resOpt & lmaxRPC3-star \\
\hline \multirow{3}{*}{$\begin{array}{l}\text { RLFAP } \\
\text { (scen, graph) }\end{array}$} & $\mathrm{t}$ & 13.5 & 61.7 & 21.7 & 26.7 & 18.8 \\
\hline & $\mathrm{n}$ & 42,250 & 8,727 & 8,727 & 7,326 & 8,727 \\
\hline & $\mathrm{cc}$ & $56 \mathrm{M}$ & $625 \mathrm{M}$ & $201 \mathrm{M}$ & $139 \mathrm{M}$ & $166 \mathrm{M}$ \\
\hline \multirow{3}{*}{$\begin{array}{l}\text { Random } \\
\text { (modelB, forced) }\end{array}$} & $\mathrm{t}$ & 2.7 & 5.8 & 3.6 & 3.9 & 3.6 \\
\hline & $\mathrm{n}$ & 29,538 & 7,385 & 7,385 & 7,835 & 7,385 \\
\hline & $\mathrm{cc}$ & $11 \mathrm{M}$ & $82 \mathrm{M}$ & $30 \mathrm{M}$ & $31 \mathrm{M}$ & $30 \mathrm{M}$ \\
\hline \multirow[t]{3}{*}{ Graph coloring } & $\mathrm{t}$ & 4.8 & 61 & 25 & 45.9 & 29.7 \\
\hline & $\mathrm{n}$ & 3,910 & 2,225 & 2,225 & 2,866 & 2,225 \\
\hline & $\mathrm{cc}$ & $12 \mathrm{M}$ & $984 \mathrm{M}$ & $284 \mathrm{M}$ & $303 \mathrm{M}$ & $283 \mathrm{M}$ \\
\hline \multirow{3}{*}{$\begin{array}{l}\text { Quasigroup } \\
\text { (qcp, qwh, bqwh) }\end{array}$} & $\mathrm{t}$ & 55.6 & 47.8 & 22 & 231.7 & 22.8 \\
\hline & $\mathrm{n}$ & 866,099 & 117,974 & 117,974 & 204,407 & 117,974 \\
\hline & $\mathrm{cc}$ & $70 \mathrm{M}$ & $315 \mathrm{M}$ & $39 \mathrm{M}$ & $71 \mathrm{M}$ & $38 \mathrm{M}$ \\
\hline \multirow[t]{3}{*}{ Geometric } & $\mathrm{t}$ & 11.3 & 50.6 & 29.1 & 30.3 & 30.1 \\
\hline & $\mathrm{n}$ & 55,825 & 26,687 & 26,687 & 27,656 & 26,687 \\
\hline & $\mathrm{cc}$ & $55 \mathrm{M}$ & $721 \mathrm{M}$ & $314 \mathrm{M}$ & $314 \mathrm{M}$ & $313 \mathrm{M}$ \\
\hline \multirow{3}{*}{$\begin{array}{l}\text { QueensKnights, } \\
\text { Queens, } \\
\text { QueenAttack }\end{array}$} & $\mathrm{t}$ & 7.1 & 133.6 & 42.7 & 56.3 & 42.5 \\
\hline & $\mathrm{n}$ & 38,663 & 4,829 & 4,829 & 22,321 & 4,829 \\
\hline & cc & $27 \mathrm{M}$ & $1,583 \mathrm{M}$ & $563 \mathrm{M}$ & $655 \mathrm{M}$ & $552 \mathrm{M}$ \\
\hline \multirow{3}{*}{$\begin{array}{l}\text { Driver, blackHole, } \\
\text { haystacks, } \\
\text { job-shop }\end{array}$} & $\mathrm{t}$ & 1.6 & 14.7 & 5.6 & 15.5 & 5.7 \\
\hline & $\mathrm{n}$ & 115,717 & 28,750 & 28,750 & 34,001 & 28,750 \\
\hline & $\mathrm{cc}$ & $3 \mathrm{M}$ & $141 \mathrm{M}$ & $33 \mathrm{M}$ & $35 \mathrm{M}$ & $32 \mathrm{M}$ \\
\hline
\end{tabular}

In Table 4 we summarize the results of our experiments by giving averages over different problem classes. These results demonstrate that 1 maxRPC $3^{r m}$ outperforms ImaxRPC ${ }^{r m}$ in all problem classes, often considerably. This was the case in all 200 instances tried. Algorithms ImaxRPC3-resOpt and especially lmaxRPC3-start display similar performance to 1 maxRPC $3^{r m}$. ImaxRPC $3-r e s O p t$ displays its worst performance in quasigroup problems where it performs twice as much constraint checks on average. Taking also into account that lmaxRPC3-resopt copies Last PC to Stop explains the variance in the results given in Tables 3 and 4. In general, ImaxRPC $3^{r m}$ is competitive with MAC on RLFAP and random instances and outperforms it on the Quasigroup classes. In contrast, 1 maxRPC $3^{r m}$ is clearly inferior to $\mathrm{AC}^{r m}$ on Queens class and in the last category that includes instances from various other structured problem classes.

\section{$6.1 d$-way branching}

We have also experimented with the above search algorithms under the $d$-way branching scheme using again the dom/wdeg heuristic for variable ordering. Table 5 reports results from the same instances as Table 3, in order to directly compare our algorithms on the two different branching schemes. We exclude lmaxRPC3-resOpt which is the less competitive among the algorithms of Table 3. We can observe that ImaxRPC $3^{r m}$ is faster by a factor of two on the RLFAP instance graph9f9, while with 2-way branching AC ${ }^{r m}$ was superior. Differences in the relative performance of $\mathrm{AC}$ and maxRPC occur in other problems as well (e.g. random, quasigroup and queensAttacking). For example, in qwh instances 1 maxRPC $3^{r m}$ has better run-time results against $\mathrm{AC}^{r m}$ but not by as large margins as under 2-way 
Table 5 Cpu times (t) in secs, nodes (n) and constraint checks (cc) from various problem instances when $d$-way branching is used

\begin{tabular}{|c|c|c|c|c|c|}
\hline Instance & & $\mathrm{AC}^{r m}$ & $\operatorname{lmaxRPC}^{r m}$ & $\operatorname{lmaxRPC}^{r m}$ & lmaxRPC3-start \\
\hline \multirow[t]{3}{*}{ scen11-f7 } & $\mathrm{t}$ & 981.9 & 3,338 & 1,128 & 870 \\
\hline & $\mathrm{n}$ & $3,696,154$ & 552,907 & 552,907 & 552,907 \\
\hline & $\mathrm{cc}$ & $4,287 \mathrm{M}$ & $31,098 \mathrm{M}$ & $9,675 \mathrm{M}$ & $8,193 \mathrm{M}$ \\
\hline \multirow[t]{3}{*}{ graph9-f9 } & $\mathrm{t}$ & 57.1 & 85.3 & 33.3 & 30.9 \\
\hline & $\mathrm{n}$ & 273,766 & 26,276 & 26,276 & 26,276 \\
\hline & $\mathrm{cc}$ & $158 \mathrm{M}$ & $729 \mathrm{M}$ & $290 \mathrm{M}$ & $242 \mathrm{M}$ \\
\hline \multirow{3}{*}{$\begin{array}{c}\text { rand-2-40-11- } \\
414-200-30\end{array}$} & $\mathrm{t}$ & 11.3 & 33.4 & 26.9 & 21.5 \\
\hline & $\mathrm{n}$ & 110,091 & 49,100 & 49,100 & 49,100 \\
\hline & $\mathrm{cc}$ & $51 \mathrm{M}$ & $484 \mathrm{M}$ & $189 \mathrm{M}$ & $187 \mathrm{M}$ \\
\hline \multirow[t]{3}{*}{ will199GPIA-6 } & $\mathrm{t}$ & 3 & 11.7 & 4.8 & 5.1 \\
\hline & $\mathrm{n}$ & 13,243 & 4,971 & 4,971 & 4,971 \\
\hline & $\mathrm{cc}$ & $13 \mathrm{M}$ & $108 \mathrm{M}$ & $42 \mathrm{M}$ & $41 \mathrm{M}$ \\
\hline \multirow[t]{3}{*}{ qcp150-120-5 } & $\mathrm{t}$ & 15.9 & 34.8 & 17.8 & 18.6 \\
\hline & $\mathrm{n}$ & 233,311 & 100,781 & 100,781 & 100,781 \\
\hline & $\mathrm{cc}$ & $27 \mathrm{M}$ & $330 \mathrm{M}$ & $54 \mathrm{M}$ & $53 \mathrm{M}$ \\
\hline \multirow[t]{3}{*}{ qcp150-120-9 } & $\mathrm{t}$ & 66.5 & 162.9 & 78.4 & 81.2 \\
\hline & $\mathrm{n}$ & $2,437,173$ & 627,679 & 627,679 & 627,679 \\
\hline & $\mathrm{cc}$ & $157 \mathrm{M}$ & $1,060 \mathrm{M}$ & $163 \mathrm{M}$ & $162 \mathrm{M}$ \\
\hline \multirow[t]{3}{*}{ qwh20-166-1 } & $\mathrm{t}$ & 14.6 & 28.2 & 15.7 & 16.4 \\
\hline & $\mathrm{n}$ & 234,095 & 54,286 & 54,286 & 54,286 \\
\hline & $\mathrm{cc}$ & $19 \mathrm{M}$ & $156 \mathrm{M}$ & $18 \mathrm{M}$ & $18 \mathrm{M}$ \\
\hline \multirow[t]{3}{*}{ qwh20-166-6 } & $\mathrm{t}$ & 462.7 & 674.3 & 346.2 & 367 \\
\hline & $\mathrm{n}$ & $4,651,632$ & 919,861 & 919,861 & 919,861 \\
\hline & $\mathrm{cc}$ & $633 \mathrm{M}$ & $5,089 \mathrm{M}$ & $566 \mathrm{M}$ & $558 \mathrm{M}$ \\
\hline \multirow[t]{3}{*}{ qwh20-166-7 } & $\mathrm{t}$ & 30 & 51.9 & 27.8 & 29.1 \\
\hline & $\mathrm{n}$ & 263,713 & 76,624 & 76,624 & 76,624 \\
\hline & $\mathrm{cc}$ & $42 \mathrm{M}$ & $392 \mathrm{M}$ & $45 \mathrm{M}$ & $44 \mathrm{M}$ \\
\hline \multirow[t]{3}{*}{ geo50-20-d4-75-1 } & $\mathrm{t}$ & 38.7 & 144.8 & 81.9 & 82.3 \\
\hline & $\mathrm{n}$ & 181,560 & 79,691 & 79,691 & 79,691 \\
\hline & $\mathrm{cc}$ & $192 \mathrm{M}$ & $2,045 \mathrm{M}$ & $880 \mathrm{M}$ & $876 \mathrm{M}$ \\
\hline \multirow[t]{3}{*}{ queenAttacking6 } & $\mathrm{t}$ & 54.7 & 406.8 & 153.9 & 146.6 \\
\hline & $\mathrm{n}$ & 262,087 & 103,058 & 103,058 & 103,058 \\
\hline & $\mathrm{cc}$ & $211 \mathrm{M}$ & $6,035 \mathrm{M}$ & $1,640 \mathrm{M}$ & $1,623 \mathrm{M}$ \\
\hline queensKnights- & $\mathrm{t}$ & 18.2 & 82.6 & 40.4 & 23.5 \\
\hline \multirow[t]{2}{*}{$15-5-\mathrm{mul}$} & $\mathrm{n}$ & 35,445 & 13,462 & 13,462 & 13,462 \\
\hline & $\mathrm{cc}$ & $154 \mathrm{M}$ & $963 \mathrm{M}$ & $387 \mathrm{M}$ & $282 \mathrm{M}$ \\
\hline \multirow[t]{3}{*}{ haystacks-05 } & $\mathrm{t}$ & 0.7 & 0.7 & 0.8 & 0.7 \\
\hline & $\mathrm{n}$ & 110,638 & 20,278 & 20,278 & 20,278 \\
\hline & $\mathrm{cc}$ & $1.4 \mathrm{M}$ & $1.9 \mathrm{M}$ & $1.1 \mathrm{M}$ & $1.0 \mathrm{M}$ \\
\hline
\end{tabular}

branching. In comparison to 1 maxRP $C^{r m}$, 1 maxRPC $3^{r m}$ remains advantageous in all instances.

Table 6 summarizes results from the application of lmaxRPC during search using $d$-way branching. We give average results for all the tested instances, grouped into specific problem classes, as in Table 4. As can be seen, ImaxRPC $3^{r m}$ and lmaxRPC3-start improve on the existing best algorithm considerably, making ImaxRPC outperform MAC on the quasigroup problem classes and be quite competitive on the RLFAP class. As expected, when comparing the same (AC or maxRPC) 
Table 6 Mean search performance in all 200 instances grouped by class, when $d$-way branching is used

\begin{tabular}{|c|c|c|c|c|c|}
\hline \multirow{4}{*}{$\begin{array}{l}\text { Problem class } \\
\text { RLFAP } \\
\text { (scen, graph) }\end{array}$} & & $\mathrm{AC}^{r m}$ & $\operatorname{lmaxRPC}^{r m}$ & $\operatorname{lmaxRPC} 3^{r m}$ & ImaxRPC3-start \\
\hline & $\mathrm{t}$ & 124 & 157.3 & 157.3 & 123.2 \\
\hline & $\mathrm{n}$ & 424,128 & 74,083 & 73,083 & 73,083 \\
\hline & $\mathrm{cc}$ & $559 \mathrm{M}$ & $4,394 \mathrm{M}$ & $1,387 \mathrm{M}$ & $1,092 \mathrm{M}$ \\
\hline \multirow{3}{*}{$\begin{array}{l}\text { Random } \\
\qquad \text { (modelB, forced) }\end{array}$} & $\mathrm{t}$ & 2.2 & 7.7 & 5.3 & 4.8 \\
\hline & $\mathrm{n}$ & 19,809 & 9,270 & 9,270 & 9,270 \\
\hline & $\mathrm{cc}$ & $9 \mathrm{M}$ & $110 \mathrm{M}$ & $40 \mathrm{M}$ & $40 \mathrm{M}$ \\
\hline \multirow[t]{3}{*}{ Graph coloring } & $\mathrm{t}$ & 8.9 & 110.3 & 46.9 & 43.4 \\
\hline & $\mathrm{n}$ & 5,919 & 2,983 & 2,983 & 2,983 \\
\hline & $\mathrm{cc}$ & $23 \mathrm{M}$ & $1,735 \mathrm{M}$ & $455 \mathrm{M}$ & $454 \mathrm{M}$ \\
\hline \multirow{3}{*}{$\begin{array}{l}\text { Quasigroup } \\
\text { (qcp,qwh,bqwh) }\end{array}$} & $\mathrm{t}$ & 35.5 & 57.4 & 29.6 & 31.2 \\
\hline & $\mathrm{n}$ & 387,495 & 103,994 & 103,994 & 103,994 \\
\hline & $\mathrm{cc}$ & $51 \mathrm{M}$ & $458 \mathrm{M}$ & $56 \mathrm{M}$ & $55 \mathrm{M}$ \\
\hline \multirow[t]{3}{*}{ Geometric } & $\mathrm{t}$ & 8.2 & 29.7 & 17.2 & 17.3 \\
\hline & $\mathrm{n}$ & 39,879 & 17,273 & 17,273 & 17.273 \\
\hline & $\mathrm{cc}$ & $39 \mathrm{M}$ & $418 \mathrm{M}$ & $180 \mathrm{M}$ & $179 \mathrm{M}$ \\
\hline \multirow{3}{*}{$\begin{array}{l}\text { QueensKnights, } \\
\text { Queens, } \\
\text { QueenAttack }\end{array}$} & $\mathrm{t}$ & 14.7 & 206.1 & 73.6 & 67.6 \\
\hline & $\mathrm{n}$ & 67,019 & 24,859 & 24,859 & 24,859 \\
\hline & $\mathrm{cc}$ & $73 \mathrm{M}$ & $2,796 \mathrm{M}$ & $839 \mathrm{M}$ & $807 \mathrm{M}$ \\
\hline \multirow{3}{*}{$\begin{array}{l}\text { Driver,blackHole, } \\
\text { haystacks, } \\
\text { job-shop }\end{array}$} & $\mathrm{t}$ & 0.8 & 13.2 & 5.2 & 5.3 \\
\hline & $\mathrm{n}$ & 13,075 & 11,349 & 11,349 & 11,349 \\
\hline & $\mathrm{cc}$ & $1 \mathrm{M}$ & $121 \mathrm{M}$ & $25 \mathrm{M}$ & $25 \mathrm{M}$ \\
\hline
\end{tabular}

algorithm under the two different branching schemes, 2-way branching is typically superior.

Overall, our results demonstrate that the efficient application of a maxRPC approximation throughout search can give an algorithm that is quite competitive with MAC on some classes of binary CSPs with either of the two standard branching schemes. This confirms the conjecture of [15] about the potential of maxRPC as an alternative to AC. In addition, our results, along with ones in [28], show that approximating strong and complex local consistencies can be very beneficial.

\subsection{Heuristics}

We have also run experiments to evaluate several of the heuristics described in Section 5. In these experiments we have mainly used the best algorithm, ImaxRPC $3^{r m}$, under 2-way braching. Intuitively, the use of heuristics may improve the algorithm's performance as explained in Section 5. Since only light versions of maxRPC are practical for use during search, we have only tested heuristics H1, $\mathrm{H} 2$ and $\mathrm{H} 3$. Recall that heuristics $\mathrm{H} 4$ and $\mathrm{H} 5$ are not applicable for light maxRPC algorithms.

With respect to the specific strategy for ordering variables under the different heuristics, we have tried all the "fail-first" methods analyzed in Section 5 (i.e. dom, del_ratio, wdeg, dom/wdeg). dom and wdeg were not as efficient as the other methods and are thus ommitted from Table 7. The algorithm used is lmaxRPC $3^{r m}$, except from the last column where we report results from lmaxRPC3-start. Apart 


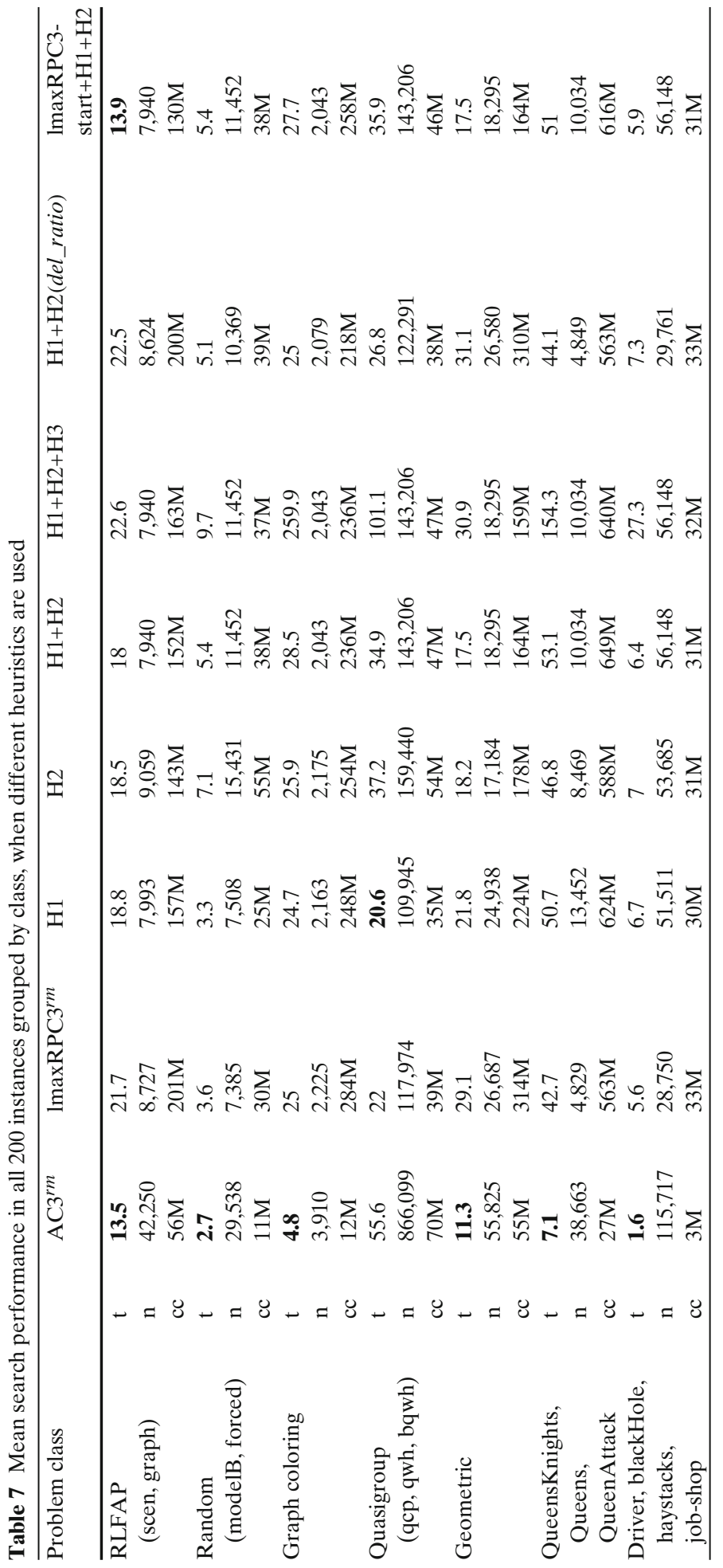


from column $H 1+H 2($ del_ratio), where the heuristic is mentioned explicitly, in the rest of the columns we use dom/wdeg.

Considering the results in Table 7 compared to results in Table 4 it seems that the application of heuristics does not offer any benefits as the algorithm's performance is marginally improved, if at all. In some problem classes using no heuristic at all is the best choice. These results, obtained using 2-way branching, are in contrast to results from [1] where it was shown that heuristics $\mathrm{H} 1$ and $\mathrm{H} 2$ are mildly beneficial when $d$-way branching is used.

The most promising is the application of both $\mathrm{H} 1$ and $\mathrm{H} 2(\mathrm{H} 1+\mathrm{H} 2)$, where $x_{j}$ extracted from $Q$ and $x_{i}$, which is the neighbouring variable of $x_{j}$, are ordered in ascending order of the dom/wdeg value. The less efficient combination is the $\mathrm{H} 1+\mathrm{H} 2+\mathrm{H} 3$ because of the run-time overhead caused by the often computation of all three heuristics. Comparing del_ratio and dom/wdeg on $\mathrm{H} 1+\mathrm{H} 2$ we conclude that the former is preferable on Quasigroup and Queen problems while the latter is better on RLFAP and Geometric problems. On the rest of the problem classes they display similar performance.

\subsection{Interleaving AC and maxRPC}

Since there are problem classes where either an algorithm that maintains AC or one that maintains lmaxRPC is preferable, we have experimented with hybrid propagation schemes that interleave $l \operatorname{maxRPC} 3^{r m}$ and $A C 3^{r m}$. Specifically, we have considered the following simple ways to interleave the two algorithms under 2-way

Table 8 Mean hybrid search performance in all 200 instances grouped by class

\begin{tabular}{|c|c|c|c|c|c|}
\hline \multirow[t]{2}{*}{ Problem class } & & \multirow[t]{2}{*}{$\mathrm{AC} 3^{r m}$} & \multirow[t]{2}{*}{$\operatorname{lmaxRPC}^{r m}$} & \multicolumn{2}{|l|}{$(x=a) \wedge(x \neq a)$} \\
\hline & & & & $\operatorname{lmaxRPC}^{r m} \wedge \mathrm{AC}^{r m}$ & $\mathrm{AC}^{r m} \wedge \operatorname{lmaxRPC}^{r m}$ \\
\hline \multirow{3}{*}{$\begin{array}{l}\text { RLFAP } \\
\text { (scen,graph) }\end{array}$} & $\mathrm{t}$ & 13.5 & 21.7 & 21.9 & 19.5 \\
\hline & $\mathrm{n}$ & 42,250 & 8,727 & 17,231 & 25,748 \\
\hline & $\mathrm{cc}$ & $56 \mathrm{M}$ & $201 \mathrm{M}$ & $193 \mathrm{M}$ & $103 \mathrm{M}$ \\
\hline \multirow{3}{*}{$\begin{array}{l}\text { Random } \\
\text { (modelB,forced) }\end{array}$} & $\mathrm{t}$ & 2.7 & 3.6 & 3.2 & 4.1 \\
\hline & $\mathrm{n}$ & 29,538 & 7,385 & 11,488 & 16,668 \\
\hline & $\mathrm{cc}$ & $11 \mathrm{M}$ & $30 \mathrm{M}$ & $24 \mathrm{M}$ & $30 \mathrm{M}$ \\
\hline \multirow[t]{3}{*}{ Graph coloring } & $\mathrm{t}$ & 4.8 & 25 & 21.6 & 5.9 \\
\hline & $\mathrm{n}$ & 3,910 & 2,225 & 2,745 & 2,654 \\
\hline & $\mathrm{cc}$ & $12 \mathrm{M}$ & $284 \mathrm{M}$ & $240 \mathrm{M}$ & $58 \mathrm{M}$ \\
\hline \multirow{3}{*}{$\begin{array}{l}\text { Quasigroup } \\
\text { (qcp,qwh,bqwh) }\end{array}$} & $\mathrm{t}$ & 55.6 & 22 & 30.2 & 40.9 \\
\hline & $\mathrm{n}$ & 866,099 & 117,974 & 233,919 & 324,373 \\
\hline & $\mathrm{cc}$ & $70 \mathrm{M}$ & $39 \mathrm{M}$ & $43 \mathrm{M}$ & $60 \mathrm{M}$ \\
\hline \multirow[t]{3}{*}{ Geometric } & $\mathrm{t}$ & 11.3 & 29.1 & 16.6 & 15.3 \\
\hline & $\mathrm{n}$ & 55,825 & 26,687 & 25,042 & 28,785 \\
\hline & $\mathrm{cc}$ & $55 \mathrm{M}$ & $314 \mathrm{M}$ & $164 \mathrm{M}$ & $140 \mathrm{M}$ \\
\hline \multirow{3}{*}{$\begin{array}{l}\text { QueensKnights, } \\
\text { Queens, } \\
\text { QueenAttack }\end{array}$} & $\mathrm{t}$ & 7.1 & 42.7 & 42.2 & 9.9 \\
\hline & $\mathrm{n}$ & 38,663 & 4,829 & 9,645 & 11,648 \\
\hline & $\mathrm{cc}$ & $27 \mathrm{M}$ & $563 \mathrm{M}$ & $535 \mathrm{M}$ & $211 \mathrm{M}$ \\
\hline \multirow{3}{*}{$\begin{array}{l}\text { Driver, blackHole } \\
\text { haystacks, job-shop }\end{array}$} & $\mathrm{t}$ & 1.6 & 5.6 & 2.1 & 1.8 \\
\hline & $\mathrm{n}$ & 115,717 & 28,750 & 64,891 & 86,446 \\
\hline & $\mathrm{cc}$ & $3 \mathrm{M}$ & $33 \mathrm{M}$ & $30 \mathrm{M}$ & $31 \mathrm{M}$ \\
\hline
\end{tabular}


branching: At any left branch we run 1 maxRPC $3^{r m}$ (respectively $\mathrm{AC}^{\mathrm{rm}}$ ) after a value assigment, while at any right branch we run $A C 3^{r m}$ (respectively 1 maxRPC $3^{r m}$ ) after a value removal. Table 8 summarizes the results of our experiments with these methods.

Given the results in Table 8, the first observation we can make is that none of the two hybrid propagation schemes is substantially worse than both 1 maxRPC $3^{\mathrm{rm}}$ and $A C 3^{r m}$ on any problem class. In contrast, there are problem classes where the hybrids outperform either maxRPC (e.g. geometric) or AC (quasigroups) by substantial margins. This means that, as expected, the hybrid methods achieve a compromise between maxRPC and AC, which is evident by looking at both cpu times and node visits. Applying maxRPC at left branches results in performance closer to maintaining maxRPC, while when AC is applied at left branches the performance is closer to MAC. This is not surprising since the effects of constraint propagation are stronger after variable assignments compared to value removals. Therefore, the local consistency applied at left branches is the "dominant" one that determines the behaviour of the algorithm. As a result, the former hybrid method is better on quasigroup problems but worse on graph coloring and queens instances, while the two are close on the rest of the problem classes.

The preliminary results presented here give a strong indication that interleaving $\mathrm{AC}$ and stronger local consistencies, such as maxRPC, during search can be quite beneficial. Further research is certainly required to develop more informed and efficient ways of interleaving different local consistencies.

\section{Conclusion}

Although maxRPC has been identified as a promising strong local consistency for binary constraints, it has received rather narrow attention since it was introduced. Only two new algortihms have been proposed since the introduction of maxRPC1, the first algorithm for maxRPC, and they have only been evaluated on random problems, if at all.

In this paper we have identified sources of redundancies in the existing maxRPC algorithms which largely contribute to the high cost of maintaining maxRPC during search. Based on this, we presented new algorithms for maxRPC, and their light versions that approximate maxRPC. These algorithms build on and improve existing maxRPC algorithms, achieving the elimination of many redundant constraint checks. We also investigated heuristics that can be used to order certain operations within maxRPC algorithms.

Experimental results from various problem classes demonstrate that our best method, lmaxRPC $3^{r m}$, constantly outperforms existing algorithms, often by large margins. Significantly, the speed-ups obtained allow lmaxRPC $3^{\mathrm{rm}}$ to compete with and outperform MAC on some problems, justifying the conjecture of [15] about the potential of maxRPC as an alternative to AC.

In the future it would be interesting to investigate the applicability of similar methods to efficiently achieve or approximate other local consistencies related to maxRPC such as PIC or maxRPCEn. Also, a very interesting direction is the efficient interleaved application of stronger consistencies, like maxRPC, and weaker but 
cheaper ones, like AC. We have presented some initial results towards this, but further research is certainly required.

Acknowledgements We would like to thank the anonymous reviewers for their insightful comments that helped improve this paper.

\section{References}

1. Balafoutis, T., Paparrizou, A., Stergiou, K., \& Walsh, T. (2010). Improving the performance of maxRPC. In Proceedings of CP-2010 (pp. 69-83).

2. Balafoutis, T., \& Stergiou, K. (2008). Exploiting constraint weights for revision ordering in arc consistency algorithms. In ECAI-08 workshop on modeling and solving problems with constraints.

3. Bartak, R., \& Erben, R. (2004). A new algorithm for singleton arc consistency. In Proceedings of FLAIRS conference-2004.

4. Berlandier, P. (1995). Improving domain filtering using restricted path consistency. In Proceedings of IEEE CAIA-95 (pp. 32-37).

5. Bessiere, C. (1994). Arc-consistency and arc-consistency again. Artificial Intelligence, 65, 179190.

6. Bessiere, C., Cardon, S., Debruyne, R., \& Lecoutre, C. (2011). Efficient algorithms for singleton arc consistency. Constraints, 16, 25-53.

7. Bessiere, C., \& Debruyne, R. (2005). Optimal and suboptimal singleton arc consistency algorithms. In Proceedings of IJCAI-2005 (pp. 54-59).

8. Bessière, C., Freuder, E. C., \& Régin, J. C. (1995). Using inference to reduce arc consistency computation. In Proceedings of IJCAI'95 (pp. 592-599).

9. Bessiere, C., Katsirelos, G., Narodytska, N., Quimper, C. G., \& Walsh, T. (2009). Decompositions of all different, global cardinality and related constraints. In Proceedings of IJCAI-2009 (pp. 419424).

10. Bessière, C., Régin, J. C., Yap, R., \& Zhang, Y. (2005). An optimal coarse-grained arc consistency algorithm. Artificial Intelligence, 165(2), 165-185.

11. Boussemart, F., Hemery, F., \& Lecoutre, C. (2004). Revision ordering heuristics for the constraint satisfaction problem. In CP-2004 workshop on constraint propagation and implementation, Toronto, Canada.

12. Boussemart, F., Hemery, F., Lecoutre, C., \& Sais, L. (2004). Boosting systematic search by weighting constraints. In Proceedings of ECAI-2004 (pp. 482-486). Valencia, Spain.

13. Debruyne, R. (1999). A strong local consistency for constraint satisfaction. In Proceedings of ICTAI-99 (pp. 202-209).

14. Debruyne, R., \& Bessière, C. (1997). From restricted path consistency to max-restricted path consistency. In Proceedings of CP-97 (pp. 312-326).

15. Debruyne, R., \& Bessière, C. (2001). Domain filtering consistencies. Journal of Artificial Intelligence Research, 14, 205-230.

16. Freuder, E., \& Elfe, C. (1996). Neighborhood inverse consistency preprocessing. In Proceedings of AAAI'96 (pp. 202-208).

17. Gent, I. P., MacIntyre, E., Prosser, P., Shaw, P., \& Walsh, T. (1997). The constraindedness of arc consistency. In Proceedings of CP-97 (pp. 327-340).

18. Grandoni, F., \& Italiano, G. (2003). Improved algorithms for max-restricted path consistency. In Proceedings of CP'03 (pp. 858-862).

19. Haralick, R. M., \& Elliott, G. L. (1980). Increasing tree search efficiency for constraint satisfaction problems. Artificial Intelligence, 14, 263-314.

20. Lecoutre, C., \& Cardon, S. (2005). A greedy approach to establish singleton arc consistency. In Proceedings of IJCAI-2005 (pp. 199-204).

21. Lecoutre, C., Cardon, S., \& Vion, J. (2007). Conservative dual consistency. In Proceedings of AAAI-07 (pp. 237-242).

22. Lecoutre, C., \& Hemery, F. (2007). A study of residual supports in arc consistency. In Proceedings of IJCAI-2007 (pp. 125-130).

23. Likitvivatanavong, C., Zhang, Y., Bowen, J., Shannon, S., \& Freuder, E. (2007). Arc consistency during search. In Proceedings of IJCAI-2007 (pp. 137-142). 
24. Montanari, U. (1974). Network of constraints: Fundamental properties and applications to picture processing. Information Science, 7, 95-132.

25. Quimper, C. G., \& Walsh, T. (2006). Global grammar constraints. In Proceedings of CP-2006 (pp. 751-755).

26. Sabin, D., \& Freuder, E. C. (1997). Understanding and improving the MAC algorithm. In Proceedings of CP-1997 (pp. 167-181).

27. Schulte, C., \& Stuckey, P. J. (2008). Efficient constraint propagation engines. ACM Transactions on Programming Languages and Systems, 31(1), 1-43.

28. Vion, J., \& Debruyne, R. (2009). Light algorithms for maintaining max-RPC during search. In Proceedings of SARA-2009.

29. Wallace, R., \& Freuder, E. (1992). Ordering heuristics for arc consistency algorithms. In $A I / G I / V I$ (pp. 163-169). Vancouver, British Columbia, Canada. 